\title{
Adaptive refinement for phase-field models of brittle fracture based on Nitsche's method
}

\author{
Alba Muixí · Sonia Fernández-Méndez • Antonio Rodríguez-Ferran
}

Received: date / Accepted: date

\begin{abstract}
A new adaptive refinement strategy for phasefield models of brittle fracture is proposed. The approach provides a computationally efficient solution to the high demand in spatial resolution of phase-field models. The strategy is based on considering two types of elements: $h$-refined elements along cracks, where more accuracy is needed to capture the solution, and standard elements in the rest of the domain. Continuity between adjacent elements of different type is imposed in weak form by means of Nitsche's method. The weakly imposition of continuity leads to a very local refinement in a simple way, for any degree of approximation and both in $2 \mathrm{D}$ and $3 \mathrm{D}$. The performance of the strategy is assessed for several scenarios in the quasi-static regime, including coalescence and branching of cracks in 2D and a twisting crack in 3D.
\end{abstract}

Keywords Phase-field modeling - Brittle fracture · Staggered scheme · Nitsche's method · Adaptive refinement

\section{Introduction}

Phase-field models of brittle fracture are widely used in computational fracture mechanics because crack propagation is automatically tracked with no need of additional criteria, contrarily to what happens in fracture models based on sharp representations of cracks. Moreover, they naturally handle branching and coalescence of cracks. The main drawback of using these models is the need of very fine meshes to properly approximate

A. Muixí · S. Fernández-Méndez · A. Rodríguez-Ferran (ه) Laboratori de Càlcul Numèric (LaCàN), Universitat Politècnica de Catalunya, Barcelona 08034, Spain.

E-mail: antonio.rodriguez-ferran@upc.edu the solution. The narrow bands describing cracks for small length-scale parameters imply sharp variations and, therefore, require very fine meshes $[1,4,5,25]$. Several approaches to dynamically refine the discretization as cracks propagate have been proposed in the literature. All of them are oriented to reduce the computational cost of phase-field simulations in cases in which crack paths are not known a priori. They may be classified into two categories, depending on whether the continuity between refined and nonrefined subdomains is imposed in strong form or in weak form.

Within the category of strong form continuity, Nagaraja et al [20] use the multi-level $h p$-FEM (finite element method) to dynamically refine the discretization around cracks. The mesh is $h$-refined up to a fixed depth, with an element size grading from the coarsest to the finest parts of the mesh. Also applying the multi-level $h p$-FEM, Patil et al [23] present an adaptive refinement technique in which phase-field equations are only solved in small domains containing crack tips, while cracks are sharply introduced via the eXtended FEM (X-FEM) in the rest of the domain. In these approaches, continuity of the solution between different discretizations is imposed by constraining the degrees of freedom of the richer part.

There have also been some proposals in the framework of isogeometric analysis $[12,13]$. In these works the refinement is spread from finer to coarser elements, since they assume refinements with one hanging node per element side.

On the other hand, if continuity is imposed in weak form, the resulting discretizations are very locally refined in narrow bands along cracks; no gradation of the element size or refinement level is needed, so there is no spreading of refinement. 
Noii et al [22] define two domains corresponding to refined and nonrefined regions, covered with two independent meshes, and then weakly impose continuity between them by means of Lagrange multipliers which are added as new unknowns to the system, in the so-called Global-Local approach.

The adaptive strategy we presented in [19] is also based on the weak imposition of continuity between subdomains with different approximations. Thanks to the Discontinuous Galerkin (DG) setting (more specifically, a Hybridizable DG formulation), the refinement strategy consists in defining only two types of elements, standard and refined. Continuity between neighboring elements of different type is naturally handled by the DG method, without resorting to transition elements nor hanging nodes.

Motivated by the good performance of our HDG adaptive strategy [19], we present here an alternative approach based on the more widely used continuous Galerkin (CG) formulation of the FEM.

We propose a simple strategy for automatic refinement for phase-field models, exploiting the fact that the refinement needed to capture the solution is known a priori from the length-scale parameter in the model. Using this peculiarity of the problem, we are able to simplify the implementation reducing to only two types of elements. A fixed background mesh is used during all the computation and nested refined elements are located along cracks.

The new methodology shares some features with our previous proposal in [19]: only two types of elements, standard and refined; weak imposition of continuity between elements of different type; damage threshold used as a refinement indicator. It also has various key differences. It is based on the widespread CG formulation, rather than in the more sophisticated HDG formulation; continuity is imposed in weak form by means of Nitsche's method, rather than via HDG fluxes; the approach is extended and applied to three-dimensional problems. An example of the refinement process can be seen in the YouTube video [18], for the fourth example in this paper.

Nitsche's method [21] is a well established approach in the literature to impose boundary conditions in weak form [7] and to enforce continuity between regions with nonmatching discretizations [3,14]. The method is an alternative to the use of Lagrange multipliers without additional unknowns. In Nitsche's method, the weak form of the problem is modified, introducing a scalar constant parameter whose value has to be appropiately chosen to ensure coercivity of the bilinear form. In fact, this parameter acts as a stabilization parameter, and differently from what happens in penalty methods, mod- erate values of order $\mathcal{O}\left(h^{-1}\right)$, with $h$ the element size, provide accuracy and optimal convergence.

The paper is organized as follows. First, in Section 2 , we give a brief overview of the underlying phasefield model for fracture. In Section 3, we describe the proposed refinement strategy, also commenting on the implementation. Then, in Section 4, we derive the formulation of Nitche's method for the phase-field equations. Numerical experiments to test the performance and robustness of the strategy can be found in Section 5, including branching and coalescence in 2D and a fully 3D example. The conclusions in Section 6 close the paper.

\section{The hybrid phase-field model}

We consider the hybrid phase-field model by Ambati et al [1]. Within a staggered approach, this model leads to a linear equilibrium equation while adopting a tensioncompression splitting. The idea behind the model is to incorporate the splitting into the equation modeling the crack evolution, but not into the degradation of the stress tensor.

Phase-field models are characterized by smearing the representation of cracks. Cracks are described as damaged regions in the material by means of the damage or phase-field variable, denoted by $d$. The damage field has value 0 at intact points of the material and value 1 at fully broken parts of it. The transition between both values is smooth, as sketched in Fig. 1.

In the hybrid model, the system of equations to be solved in a body occupying a domain $\Omega \subset \mathbb{R}^{n_{s d}}$, with

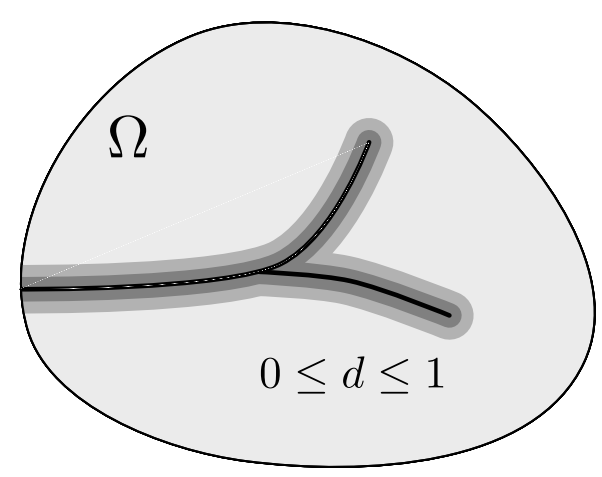

Fig. 1: Smeared representation of a crack in phase-field models. 
$n_{s d}=2,3$, reads

$$
\left\{\begin{array}{l}
\boldsymbol{\nabla} \cdot \boldsymbol{\sigma}=\mathbf{0} \text { with } \boldsymbol{\sigma}=g(d) \frac{\partial \Psi_{0}(\varepsilon)}{\partial \varepsilon}, \\
-l^{2} \Delta d+d=\frac{2 l}{G_{C}}(1-d) \mathcal{H}^{+}, \\
g(d):= \begin{cases}(1-d)^{2} & \text { where } \Psi_{0}^{+} \geq \Psi_{0}^{-}, \\
1 & \text { otherwise. }\end{cases}
\end{array}\right.
$$

Equation (1a) imposes equilibrium of forces. Here, the stress tensor $\boldsymbol{\sigma}$ is degraded by the quadratic function $g(d)$. Adding a small dimensionless parameter $\eta$ to $g(d)$ was a common practice in the first phase-field models in order to prevent a complete loss of stiffness in cracked regions. However, according to our numerical experience and some other recent works $[9,15,25]$, no artificial stiffness is needed in practice.

We restrict ourselves to the case of linear isotropic materials, for which the energy density $\Psi_{0}$ can be expressed as $\Psi_{0}(\varepsilon)=(\varepsilon: C: \varepsilon) / 2$, with $\varepsilon$ the small strain tensor and $\boldsymbol{C}$ a fourth-order tensor depending on the Lamé parameters.

Equation (1b) models the evolution of the damage field. In the equation, $G_{C}$ is the energy release rate of the material. The parameter $l$ is related to the width of smeared cracks and it is typically chosen small to approximate the behavior of sharp cracks. Thus, the value of $l$ determines the spatial discretization needed to resolve the cracks. The tension-compression splitting comes into play in the source term through the history field $\mathcal{H}^{+}$, which was introduced by Miehe et al $[16,17]$ and is defined as

$\mathcal{H}^{+}(\boldsymbol{x}, t)=\max _{\tau \in[0, t]} \Psi_{0}^{+}(\varepsilon(\boldsymbol{x}, \tau))$,

where $\Psi_{0}^{+}$denotes the tensile component of the elastic energy density, in opposition to the compressive component $\Psi_{0}^{-}$. Considering only the tensile component in the source term ensures that cracks are caused only by tension.

In this paper, we adopt the tension-compression splitting by Miehe et al $[16,17]$, based on the spectral decomposition of the strain tensor $\varepsilon$. Denoting the principal strains by $\left\{\varepsilon_{i}\right\}_{i=1, \ldots, n_{s d}}$ and the principal strain directions by $\left\{\boldsymbol{d}_{i}\right\}_{i=1, \ldots, n_{s d}}$,

$\Psi_{0}^{ \pm}(\varepsilon)=\frac{1}{2} \lambda\langle\operatorname{tr}(\varepsilon)\rangle_{ \pm}^{2}+\mu \operatorname{tr}\left(\varepsilon_{ \pm}^{2}\right)$,

where $\boldsymbol{\varepsilon}_{ \pm}=\sum_{i=1}^{n_{s d}}\left\langle\varepsilon_{i}\right\rangle_{ \pm} \boldsymbol{d}_{i} \otimes \boldsymbol{d}_{i}$ and $\langle\odot\rangle_{ \pm}=(\odot \pm|\odot|) / 2$. The definition of $\mathcal{H}^{+}$also guarantees irreversibility of cracks.

Finally, the condition in equation (1c) complements the system to ensure no interpenetration of faces occurs under compression, restoring the original stiffness of the material when compression dominates over tension. This is actually an alternative to incorporating the splitting in equation (1a). In this way, we are able to keep a linear equilibrium equation [1].

The system is solved in an incremental procedure. Given the solution at load step $n$, the solution at step $n+1$ is computed solving the system in (1) with boundary conditions

$\begin{cases}\boldsymbol{\sigma} \cdot \boldsymbol{n}=\boldsymbol{t}_{N}^{n+1} & \text { on } \Gamma_{N}, \\ \boldsymbol{u}=\boldsymbol{u}_{D}^{n+1} & \text { on } \Gamma_{D}, \\ \boldsymbol{\nabla} d \cdot \boldsymbol{n}=0 & \text { on } \partial \Omega,\end{cases}$

where $\boldsymbol{t}_{N}^{n+1}$ and $\boldsymbol{u}_{D}^{n+1}$ are the prescribed tractions and displacements, respectively, and $\boldsymbol{n}$ is the outward unit normal to the boundary. $\Gamma_{D}$ and $\Gamma_{N}$ stand for the Dirichlet and Neumann boundaries for the equilibrium equation, satisfying $\Gamma_{D} \cap \Gamma_{N}=\emptyset$ and $\partial \Omega=\Gamma_{D} \cup \Gamma_{N}$.

At each load step, we solve the system using a staggered scheme. This is, we solve the equilibrium and the damage equations alternately until convergence. The condition in (1c) is imposed in the elemental computations for the equilibrium equation using the solution from the previous staggered iteration. As a stopping criterion, we check if the error of the damage field $d$ in the Euclidean norm is lower than a fixed tolerance.

For more details on the hybrid model, as well as for an extensive review on phase-field models, we refer to Ambati et al [1].

\section{Adaptive refinement strategy}

Phase-field simulations need more resolution locally near cracks, where the displacement and damage fields present sharp variations. As cracks propagate, the space of approximation has to be accordingly refined to obtain a good representation of the solution. Here, we propose a strategy for a dynamically $h$-refined discretization. The key ingredients of our proposal are

$i$. the definition of two types of element with different approximation spaces, standard and refined, mapped into a fixed background mesh,

ii. a fixed refinement factor in refined elements, which is known a priori depending on the length-scale $l$ of the model, and

iii. the weak imposition of continuity on the interface between refined and standard elements by means of Nitsche's method.

This section aims to give a complete description of the strategy, regarding also the implementation.

In principle, the approach is also applicable to $p$ and $h p$-adaptivity. However, these options are not considered here since the sharp variations of the solution 
may cause oscillations when using a high degree $p$ for the approximation.

\subsection{The refinement process}

We consider two types of reference element: standard and refined. Standard elements are mapped to the standard reference element, whose space of approximation is the space of polynomials up to degree $p, \mathcal{P}^{p}$, as usual in a finite element approximation. Refined elements are mapped to the refined reference element, whose space of approximation is $h$-refined with a uniform submesh with $m^{n_{s d}}$ subelements, for a given refinement factor $m$. Clearly, this leads to a richer approximation space. The factor $m$ is such that the resulting discretization is able to resolve the length-scale parameter $l$ of the phase-field model.

The computational mesh describes the geometry and is fixed during all the simulation. Elements along cracks are refined, while the rest of the elements of the mesh are assumed as standard. As the simulation evolves and cracks propagate, more elements become refined. This strategy leads to a nonconformal discretization and special treatment on the interface between the two types of element is needed.

The proposed discretization is equivalent to a nonconformal $h$-refinement. The implementation with a refined reference element is chosen here for convenience, since it reduces the cases to consider to the minimum, and allows keeping the initial mesh as background mesh in the whole computation. It is worth noting that this particular refinement strategy is suited for crack tracking problems with phase-field models because the required element size in refined elements is known a priori, depending only on the length-scale $l$. It would not be applicable to adaptive refinement in other contexts.

Fig. 2 illustrates an approximation in two consecutive load steps. The discretization is $h$-refined in a narrow band containing the crack; considering only two types of element results in a very local refinement, with no spreading of the refined zone. On the interface $\Gamma$ between standard and refined elements (in red in the figure), one needs to impose continuity of the solution.

With the aim of retaining very local refinements, our choice is to impose continuity on the interface in weak form. In the case of imposing continuity in strong form, one would have to deal with the hanging nodes of the nonconformal approximation. Finding the relations between nodes for an arbitrary refinement factor $m$ may be cumbersome in practice, with several cases to implement, specially in $3 \mathrm{D}$.

In this work, we use Nitsche's method to weakly impose continuity. This method keeps the original size of the system, this is, it does not introduce extra variables. The formulation and some details of the method are presented in Section 4.

Refining criterion The damage field $d$ is a natural indicator of whether an element needs to be refined or not. An element is refined if the value of $d$ in one of its nodes reaches a fixed threshold $d^{*}$. In our numerical experience, values for $d^{*}$ around 0.2 result in accurate and robust computations.

It is important noting that some elements must be refined from the beginning where crack inception is expected, for instance at the tip of preexisting notches. Also, since brittle fracture cracks can fully grow in a single load step, the criterion has to be applied at every iteration of the staggered scheme.

Here, we do not consider derefinement of elements because a fine discretization is needed along the whole crack to ensure accuracy of the solution. An option to coarsen the discretization when the crack has already crossed some elements would be to introduce the crack as a strong discontinuity, with an X-FEM philosophy $[24,8]$.

\subsection{Refined reference element}

We define two reference elements, one for each type of element: standard and refined. Elements in the computational mesh are then mapped to the corresponding reference element depending on its type. In this way, the integration and the assembly for all elements can be done as usual. This is a viable option in this case because the refinement factor $m$ is fixed in refined elements. Therefore, the geometrical information of the $h$ refinement inside these elements is computed only once in the preprocess.

The refined reference element considers the full approximation space for each subelement, with a continuous approximation between subelements. Fig. 3 shows the discretization for a refined reference element in 1D, with degree of approximation $p=2$ and refinement factor $m=2$. The refined element is divided into two subelements. Thus, it has 5 nodes and 5 shape functions. The reference element has all the integration points of the subelements.

Refining the reference element enables to use the strategy for nonstructured meshes without any additional consideration. 

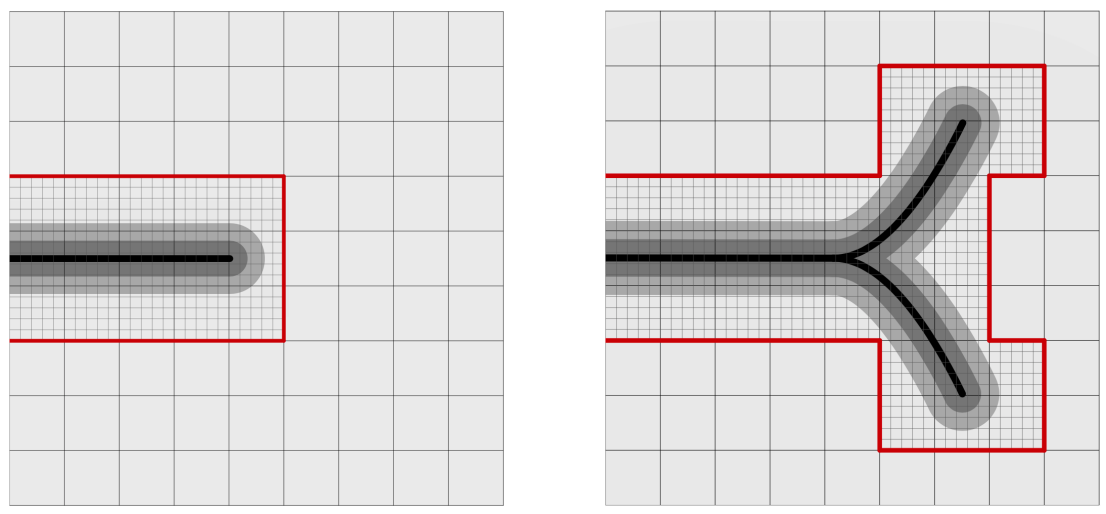

Fig. 2: Scheme of the discretization in two consecutive steps, with $h$-refined elements along the crack and standard elements in the rest of the domain. The interface on which continuity is imposed in weak form is in red.

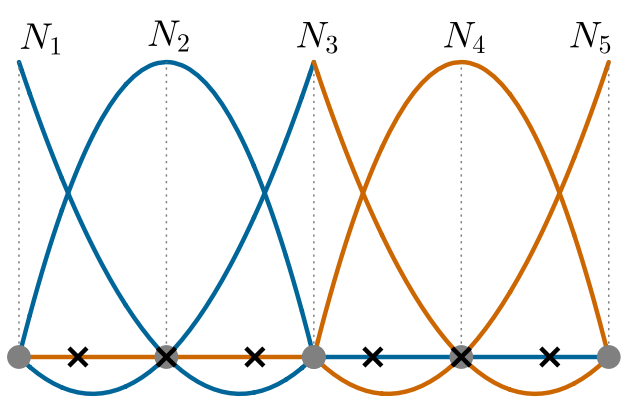

Fig. 3: Refined reference element in 1D, for degree $p=2$ and refinement factor $m=2$. Nodes are represented by grey nodes and integration points, by black crosses. The element has 5 shape functions.

\subsection{Geometrical information and update of the refined} zone

During all the simulation, information for the integration on elements and on the interface $\Gamma$ needs to be accordingly updated to account for the new refined elements.

We keep the background mesh $(X, T)$ fixed, with $X$ the nodal coordinates matrix and $T$ the connectivity matrix. The original mesh describes the geometry of the domain during all the process. The information about the refinement includes a mesh for the refined part of the domain, $\left(X_{\text {ref }}, T_{\text {ref }}\right)$, and a list of faces on the interface $\Gamma$, for which continuity is to be imposed by Nitsche's method.

The refined mesh $\left(X_{\text {ref }}, T_{\text {ref }}\right)$ is created with mappings of the refined reference element to the physical elements in the refined zone. Every time an element is refined, its subelements are added to $\left(X_{\text {ref }}, T_{\text {ref }}\right)$. This refined mesh is defined only for the assembly, using the connectivity matrix $T_{\text {ref }}$ to ensure continuity between adjacent refined elements. The numerical integration and basis functions are computed just using the integration points and basis functions in the refined reference element. Note that the isoparametric transformation can be defined using physical nodes in the background mesh $(X, T)$.

The implementation of Nitsche's method requires computing integrals on the interface between refined and nonrefined zones, $\Gamma$. To do so, as a preprocess, interior faces of the mesh $(X, T)$ are numbered and for all of them we store the number of the elements sharing the face and the local number of the face in each element, i.e., we save four integers per face. Then, during the computation, a list of the faces on the interface $\Gamma$ is updated at every iteration, accounting for the new refined elements.

\section{Nitsche's formulation}

In this section, we state the formulation of Nitsche's method for the equilibrium and the damage equations. Recall that, within the staggered scheme used to solve the phase-field system, the two formulations are independent.

For the equilibrium equation we use the formulation for linear elasticity, accounting for the damage field in the stress-strain constitutive equation. For the damage equation, we add the reaction term to the formulation for the Laplace problem. The original formulations for Nitsche's method applied to interface problems can be found in Hansbo [11].

Here, Nitsche's method is used to weakly impose continuity between subdomains with different spaces of approximation, standard and refined. Throughout the section, we denote these subdomains as $\Omega_{1}$ and $\Omega_{2}$, satisfying $\bar{\Omega}=\bar{\Omega}_{1} \cup \bar{\Omega}_{2}, \Omega_{1} \cap \Omega_{2}=\emptyset$. We define the interface where continuity is to be imposed as $\Gamma=\partial \Omega_{1} \cap \partial \Omega_{2}$, see Fig. 4 . 


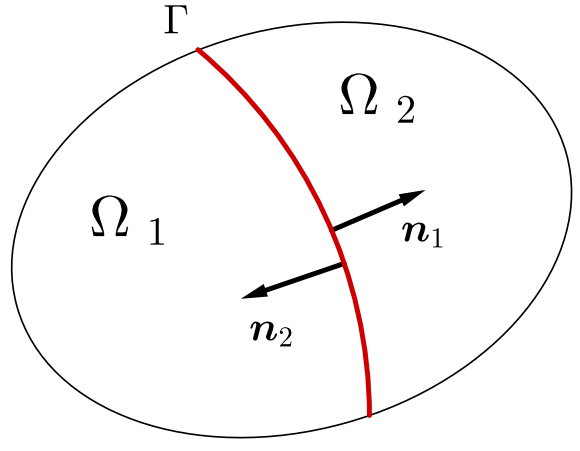

Fig. 4: Adjacent domains $\Omega_{1}$ and $\Omega_{2}$, with different approximation spaces in each one of them. Continuity is imposed by Nitsche's method on $\Gamma$ (in red).

We define the functional space

$\mathcal{V}(\Omega)=\left\{v \in L^{2}(\Omega):\left.v\right|_{\Omega_{i}} \in \mathcal{H}^{1}\left(\Omega_{i}\right)\right.$, for $\left.i=1,2\right\}$,

including discontinuous functions across $\Gamma$.

Throughout the section, the mean and jump operators are defined as $\{\odot\}=\frac{1}{2}\left(\odot_{1}+\odot_{2}\right)$ and $\llbracket \odot \rrbracket=$ $\odot_{1} \boldsymbol{n}_{1}+\odot_{2} \boldsymbol{n}_{2}=\left(\odot_{1}-\odot_{2}\right) \boldsymbol{n}_{1}$, respectively, with $\boldsymbol{n}_{1}, \boldsymbol{n}_{2}$ the unit exterior normals to $\Omega_{1}, \Omega_{2}$. Lower indices 1 and 2 on functions indicate their values on $\Gamma$ from $\Omega_{1}$ and $\Omega_{2}$, respectively.

\subsection{Equilibrium equation}

The equilibrium equation in (1a) is rewriten in the broken domain $\Omega$ as

$$
\begin{cases}\boldsymbol{\nabla} \cdot \boldsymbol{\sigma}(\boldsymbol{u}, d)=\mathbf{0} & \text { in } \Omega=\Omega_{1} \cup \Omega_{2} \\ \llbracket \boldsymbol{u} \otimes \boldsymbol{n} \rrbracket=\mathbf{0} & \text { on } \Gamma, \\ \llbracket \boldsymbol{\sigma}(\boldsymbol{u}, d) \cdot \boldsymbol{n} \rrbracket=\mathbf{0} & \text { on } \Gamma, \\ \boldsymbol{u}=\boldsymbol{u}_{D} & \text { on } \Gamma_{D}, \\ \boldsymbol{\sigma}(\boldsymbol{u}, d) \cdot \boldsymbol{n}=\boldsymbol{t}_{N} & \text { on } \Gamma_{N} .\end{cases}
$$

Equation (5a) imposes equilibrium and is complemented with the usual boundary conditions (5d) and (5e). Transmission conditions on $\Gamma$ have to be added to the system to ensure continuity of displacements, (5b), and equilibrium of tractions, $(5 \mathrm{c})$, on the interface between the two subdomains. Since the equilibrium equation is solved for a given damage field $d$ in the staggered approach, the dependence of stress $\sigma$ on $d$ is not explicitly shown in what follows.

The strategy to derive the formulation consists in writing the standard finite element weak form for each one of the subdomains, summing them, and then adding the necessary integrals to impose the extra conditions on $\Gamma$ and assure symmetry and coercivity of the bilinear form, while keeping the consistency of the formulation.

Considering the weak form in $\Omega_{1}$ and $\Omega_{2}$, separately, and summing them, we obtain that $\boldsymbol{u}$ has to satisfy

$$
\begin{aligned}
\int_{\Omega} \boldsymbol{\nabla} \boldsymbol{v}: \boldsymbol{\sigma}(\boldsymbol{u}) \mathrm{d} V-\int_{\Gamma}\left(\boldsymbol{v}_{1} \cdot \boldsymbol{\sigma}\left(\boldsymbol{u}_{1}\right) \cdot \boldsymbol{n}_{1}+\right. \\
\left.\boldsymbol{v}_{2} \cdot \boldsymbol{\sigma}\left(\boldsymbol{u}_{\mathbf{2}}\right) \cdot \boldsymbol{n}_{2}\right) \mathrm{d} s-\int_{\Gamma_{N}} \boldsymbol{v} \cdot \boldsymbol{t}_{N} \mathrm{~d} s=0,
\end{aligned}
$$

for all $\boldsymbol{v} \in[\mathcal{V}(\Omega)]^{n_{s d}}$ such that $\boldsymbol{v}=\mathbf{0}$ on $\Gamma_{D}$.

To impose condition $(5 \mathrm{c})$, we arrange the second integral in (6) by using the algebraic identity

$\boldsymbol{a}_{1} \cdot \boldsymbol{b}_{1} \cdot \boldsymbol{n}_{1}+\boldsymbol{a}_{2} \cdot \boldsymbol{b}_{2} \cdot \boldsymbol{n}_{2}=\{\boldsymbol{a}\} \cdot \llbracket \boldsymbol{b} \cdot \boldsymbol{n} \rrbracket+\llbracket \boldsymbol{a} \otimes \boldsymbol{n} \rrbracket:\{\boldsymbol{b}\}$,

which can be easily proved with the definitions of the operators. Thus, using (7) and the equilibrium of tractions on $\Gamma(5 \mathrm{c})$, equation (6) becomes

$$
\begin{aligned}
& \int_{\Omega} \boldsymbol{\nabla} \boldsymbol{v}: \boldsymbol{\sigma}(\boldsymbol{u}) \mathrm{d} V-\int_{\Gamma} \llbracket \boldsymbol{v} \otimes \boldsymbol{n} \rrbracket:\{\boldsymbol{\sigma}(\boldsymbol{u})\} \mathrm{d} s \\
& -\int_{\Gamma_{N}} \boldsymbol{v} \cdot \boldsymbol{t}_{N} \mathrm{~d} s=0 .
\end{aligned}
$$

At this step, the resulting bilinear form is neither symmetric nor coercive. Two consistent integrals, i.e. null integrals due to continuity (5b), are added to remedy these issues, leading to the weak form: find $\boldsymbol{u} \in[\mathcal{V}(\Omega)]^{n_{s d}}$ such that $\boldsymbol{u}=\boldsymbol{u}_{D}$ on $\Gamma_{D}$ and

$$
\begin{aligned}
& \int_{\Omega} \boldsymbol{\nabla} \boldsymbol{v}: \boldsymbol{\sigma}(\boldsymbol{u}) \mathrm{d} V-\int_{\Gamma} \llbracket \boldsymbol{v} \otimes \boldsymbol{n} \rrbracket:\{\boldsymbol{\sigma}(\boldsymbol{u})\} \mathrm{d} s \\
& -\int_{\Gamma}\{\boldsymbol{\sigma}(\boldsymbol{v})\}: \llbracket \boldsymbol{u} \otimes \boldsymbol{n} \rrbracket \mathrm{d} s+\beta_{\mathrm{E}} \int_{\Gamma} \llbracket \boldsymbol{u} \otimes \boldsymbol{n} \rrbracket: \llbracket \boldsymbol{v} \otimes \boldsymbol{n} \rrbracket \mathrm{d} s \\
& -\int_{\Gamma_{N}} \boldsymbol{v} \cdot \boldsymbol{t}_{N} \mathrm{~d} s=0,
\end{aligned}
$$

for all $\boldsymbol{v} \in[\mathcal{V}(\Omega)]^{n_{s d}}$ such that $\boldsymbol{v}=\mathbf{0}$ on $\Gamma_{D}$, with $\beta_{\mathrm{E}}$ a positive scalar constant. The third integral in (9) makes the functional symmetric and imposes condition (5b). The fourth integral ensures coercivity of the bilinear form for $\beta_{\mathrm{E}}$ large enough, leading to a positive definite matrix in the discrete linear system.

Assuming $\Omega_{1}$ is covered by standard elements and $\Omega_{2}$ is covered by refined elements, the discrete space for each component of the solution is

$$
\begin{array}{r}
\mathcal{V}^{h}(\Omega)=\left\{v \in \mathcal{V}(\Omega):\left.v\right|_{K_{i}} \in P^{p}\left(K_{i}\right) \text { if } K_{i} \subseteq \Omega_{1},\right. \\
\left.\left.v\right|_{K_{i}} \in P_{\text {ref }}^{p}\left(K_{i}\right) \text { if } K_{i} \subseteq \Omega_{2}\right\},
\end{array}
$$

for a finite element mesh with elements $K_{i}$, where $\mathcal{P}^{p}$ is the space of polynomials up to degree $p$ and $\mathcal{P}_{\text {ref }}^{p}\left(K_{i}\right)=\left\{v \in \mathcal{H}^{1}\left(K_{i}\right):\left.v\right|_{K_{i j}} \in \mathcal{P}^{p}\left(K_{i j}\right), j=1 \ldots m^{n_{s d}}\right\}$, 
with $K_{i j}$ the subelements from the $h$-refinement of $K_{i}$. That is, a $p$-th degree standard approximation is considered in $\Omega_{1}$ and a continuous $p$-th degree refined approximation based on subelements is considered in the regions containing the crack, $\Omega_{2}$.

The stability of the formulation depends on the value of $\beta_{\mathrm{E}}$. To obtain optimal orders of convergence $(p+1$ in $L^{2}$ norm for approximations of degree $p$ ), this parameter can be taken of the form

$\beta_{\mathrm{E}}=\alpha_{\mathrm{E}} E(h / m)^{-1}$,

with $E$ the Young's modulus and $h$ the element size in the background mesh. Taking into account this relation, the parameter that we tune is $\alpha_{\mathrm{E}}$.

It is well-known that the formulation is very robust in terms of the Nitsche's parameter. In practice, moderate values for $\alpha_{\mathrm{E}}$ are enough to ensure stability of the solution and there is a wide interval of proper values. When its value is not large enough, solutions are clearly wrong and the unstabilities can be appreciated at plain sight. Going to the other extreme, for values of $\alpha_{\mathrm{E}}$ which are much larger than the minimum value providing coercivity, the matrix becomes ill-conditioned [7].

Griebel et al [10] propose to approximate the lower bound of Nitsche's parameter by solving an eigenvalue problem. Annavarapu et al [2] show that a careful selection of this parameter is required in interfacial problems involving large material heterogeneities and/or small cut elements. This is not the case in this work, where the Nitsche's method is applied to glue nonconformal approximations. In fact, in our experience, experimentally tuning the parameter is feasible, see Section 4.4.

Notice that imposing continuity on the interface by Nitsche's method, the dimension of the resulting system does not increase.

Classical penalty methods are simpler to derive and implement, but they are based on a non-consistent weak form and need much larger parameters, of order $\mathcal{O}\left(h^{-(p+1)}\right.$ for optimal convergence. This leads to very large penalty parameters and ill-conditioning of the matrix, or inaccurate results [7].

\subsection{Damage equation}

The Nitsche formulation for the damage equation is obtained analogously to the equilibrium one. In this case, we rewrite equation (1b) as

$$
\begin{cases}-G_{C} l \Delta d+\left(\frac{G_{C}}{l}+2 \mathcal{H}^{+}\right) & \\ \qquad=2 \mathcal{H}^{+} & \text {in } \Omega=\Omega_{1} \cup \Omega_{2}, \\ \llbracket d \boldsymbol{n} \rrbracket=\mathbf{0} & \text { on } \Gamma, \\ \llbracket \boldsymbol{\nabla} d \cdot \boldsymbol{n} \rrbracket=0 & \text { on } \Gamma, \\ \boldsymbol{\nabla} d \cdot \boldsymbol{n}=0 & \text { on } \partial \Omega .\end{cases}
$$

Again, conditions in (11b) and (11c) impose continuity of the damage field and its normal derivative on the interface $\Gamma$.

The corresponding weak form reads: find $d \in \mathcal{V}(\Omega)$, such that

$$
\begin{aligned}
& \int_{\Omega}\left(\frac{G_{C}}{l}+2 \mathcal{H}^{+}\right) v d \mathrm{~d} V+\int_{\Omega} G_{C} l \nabla v \cdot \nabla d \mathrm{~d} V \\
& -\int_{\Gamma} G_{C} l \llbracket v \boldsymbol{n} \rrbracket \cdot\{\boldsymbol{\nabla} d\} \mathrm{d} s-\int_{\Gamma} G_{C} l \llbracket d \boldsymbol{n} \rrbracket \cdot\{\boldsymbol{\nabla} v\} \mathrm{d} s \\
& +\beta_{\mathrm{D}} \int_{\Gamma} \llbracket d \boldsymbol{n} \rrbracket \cdot \llbracket v \boldsymbol{n} \rrbracket \mathrm{d} s=\int_{\Omega} v 2 \mathcal{H}^{+} \mathrm{d} V,
\end{aligned}
$$

for all $v \in \mathcal{V}(\Omega)$ and with $\beta_{\mathrm{D}}$ a sufficiently large scalar parameter. To obtain optimal convergence, the Nitsche's parameter can be taken as

$\beta_{\mathrm{D}}=\alpha_{\mathrm{D}} G_{C} l(h / m)^{-1}$,

with $\alpha_{\mathrm{D}}$ to be tuned or determined from an eigenvalue problem [10].

\subsection{Convergence of the formulation}

We study the convergence of the previous formulations with respect to an analytical solution, both in $2 \mathrm{D}$ and 3D. In all cases, the Nitsche's parameter is $\alpha=100$. In the convergence plots, $h$ refers to the element size of the background mesh and the numbers correspond to the slope in each segment.

Convergence in 2D. Consider the domain $\Omega=[0,1]^{2}$, with a refined approximation in elements in $[0,0.5] \times$ [0, 1], with refinement factor $m=4$, and a standard approximation in elements in $[0.5,1] \times[0,1]$. Continuity on $\Gamma=\{x=0.5\} \cap \Omega$ is imposed using Nitsche's method. We study the convergence when refining the background mesh, maintaining $m$ fixed. Fig. 5 shows the coarsest discretization for degree $p=2$.

For the equilibrium equation, we set the source term and Dirichlet boundary conditions on $\partial \Omega$ such that the analytical solution is

$\boldsymbol{u}(x, y)=\left(\begin{array}{c}\sin (3 x+y) \\ \cos (x+3 y)\end{array}\right)$ 


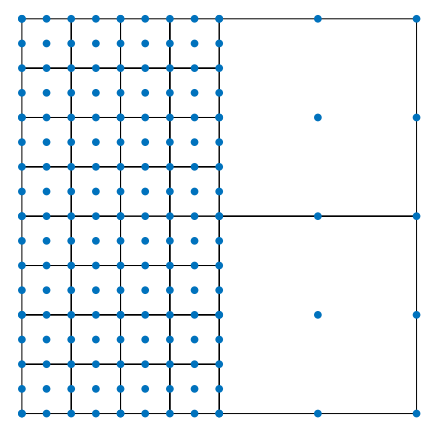

Fig. 5: Convergence in 2D. Coarsest discretization in $\Omega$ for degree $p=2$ and refinement factor $m=4$. Blue dots indicate the nodes.

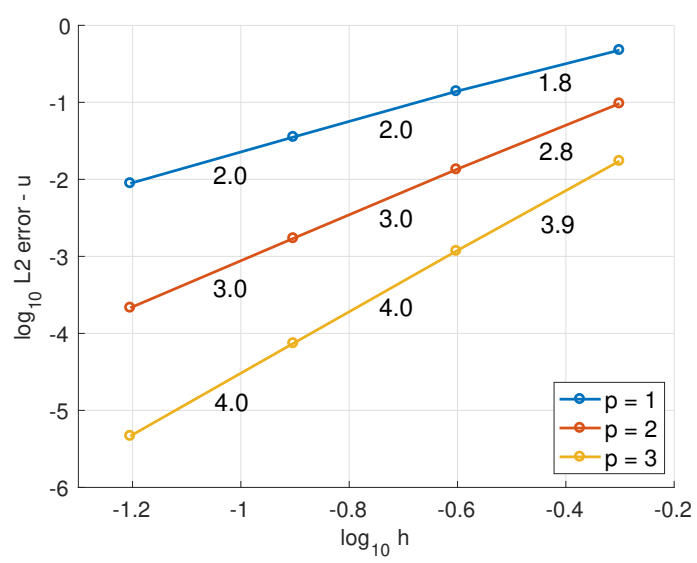

Fig. 6: Equilibrium equation in 2D. Convergence plot of the displacement $\boldsymbol{u}$, for degrees $p=1,2,3$ and $\alpha_{\mathrm{E}}=$ 100.

with $d(x, y)=\frac{\sin (x+y)+1}{5}$. The parameters are $E=$ $20 \mathrm{GPa}$ and $\nu=0.18$. Fig. 6 shows the convergence plot in this case for degrees of approximation $p$, in agreement with the theoretical orders.

For the damage equation, the boundary conditions and the source term $\mathcal{H}^{+}$are set accordingly to the analytical solution

$$
d(x, y)=\frac{\sin (3 x+y)+1}{3},
$$

with $G_{C}=2.7 \cdot 10^{-3} \mathrm{kN} / \mathrm{mm}$ and $l=0.01 \mathrm{~mm}$. Convergence plots are depicted in Fig. 7, again exhibiting optimal orders of convergence.

Convergence in 3D. Analogously, we now consider the domain $\Omega=[0,1]^{3}$, which is discretized with refined elements for $\{x<0.5\}$ and with standard elements for $\{x>0.5\}$. Thus, $\Gamma=\{x=0.5\} \cap \Omega$.

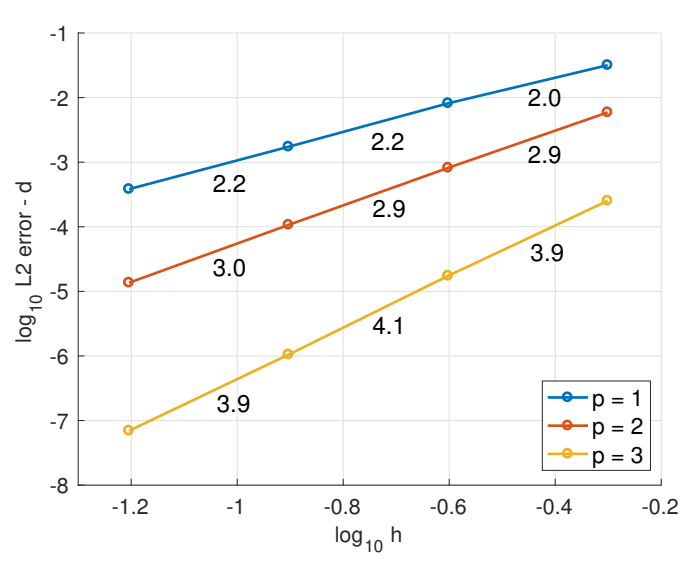

Fig. 7: Damage equation in 2D. Convergence plot for the damage $d$, for degrees $p=1,2,3$ and $\alpha_{\mathrm{D}}=100$.

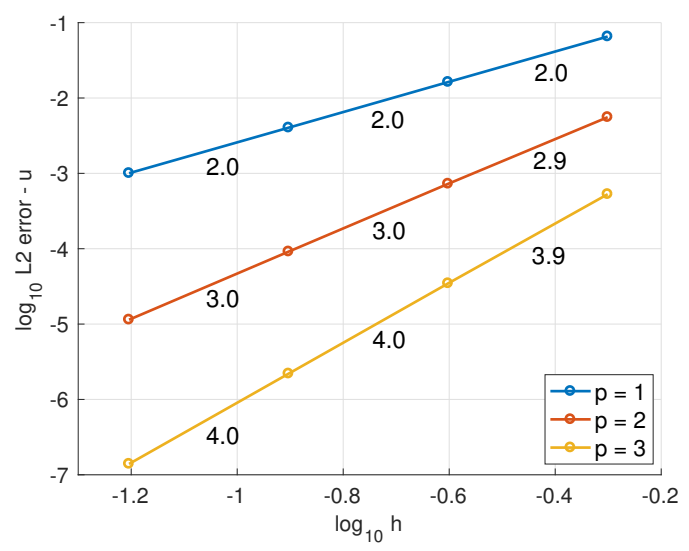

Fig. 8: Equilibrium equation in 3D. Convergence plot of the displacement $\boldsymbol{u}$, for degrees $p=1,2,3$ and $\alpha_{\mathrm{E}}=$ 100. is

For the equilibrium equation, the analytical solution

$$
\boldsymbol{u}(x, y, z)=\left(\begin{array}{c}
\sin (3 y+z) \\
\sin (x+3 z) \\
x^{6}+2
\end{array}\right)
$$

with $d(x, y)=\frac{\sin (x+y+z)+1}{5}, E=20 \mathrm{GPa}$ and $\nu=$ 0.18 . The expected orders of convergence are obtained, as can be seen in Fig. 8 .

For the damage equation, we study the convergence to the solution

$$
d(x, y, z)=\frac{\sin (3 x+2 y+z)+1}{3}
$$

with $G_{C}=2.7 \cdot 10^{-3} \mathrm{kN} / \mathrm{mm}$ and $l=0.01 \mathrm{~mm}$. Results are displayed in Fig. 9, showing optimal convergence. 


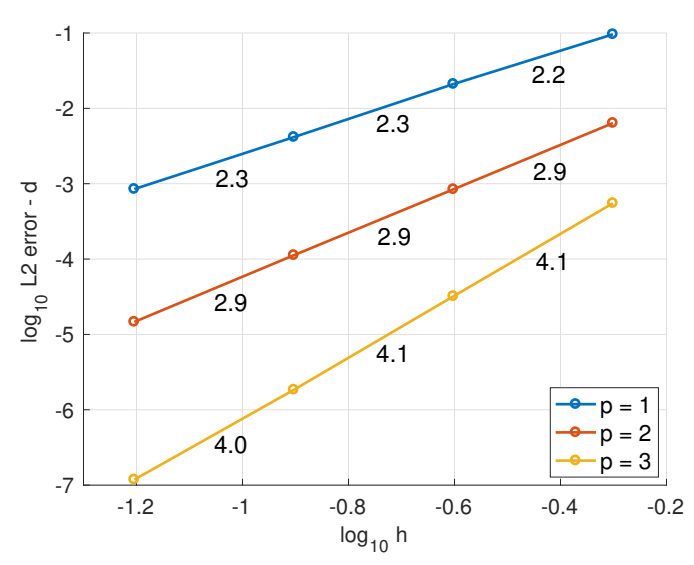

Fig. 9: Damage equation in 3D. Convergence plot for the damage $d$, for degrees $p=1,2,3$ and $\alpha_{\mathrm{D}}=100$.

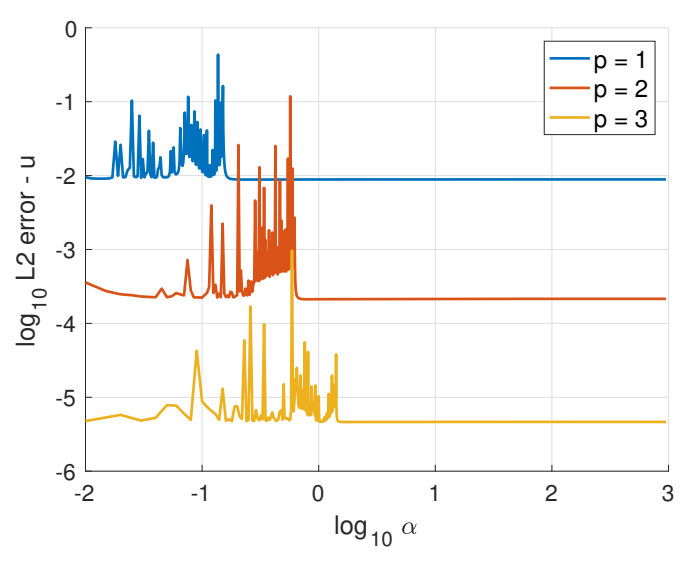

Fig. 10: Equilibrium equation in 2D. Error of the formulation for different values of Nitsche's parameter $\alpha_{\mathrm{E}}$.

\subsection{Choice of Nitsche's parameter}

The effect of parameter $\alpha$ in the Nitsche's formulations is explored next. The good behavior of the method with respect to this parameter enables to easily choose a proper value. We focus on the equilibrium equation in 2D. However, the conclusions are extendable to other cases.

Assume the parameters and the analytical solution for the convergence test in (14). The domain is discretized with the third mesh, with element size $h=$ 0.125 , refining elements in $\{x<0.5\}$ with refinement factor $m=4$. Fig. 10 shows the variations in $L^{2}$ error for values of $\alpha_{\mathrm{E}} \in\left[10^{-2}, 10^{3}\right]$, for degrees $p=1,2,3$.

For all degrees, we observe that there is a critical value $\alpha_{\min }^{p}$ such that the solution is stable for any $\alpha>$ $\alpha_{\min }^{p}$. Moreover, the plots also show that for $\alpha>\alpha_{\min }^{p}$ the accuracy does not depend on the particular value of $\alpha$, exhibiting a very robust behavior on the parame- ter. On the other hand, values of $\alpha$ below this critical value provide solutions that are clearly wrong just by visual inspection, making the tuning of $\alpha$ an easy task. Note that the critical value increases with the degree of approximation. As a safe value, we take $\alpha=100$ in all simulations.

\section{Numerical experiments}

In this section, we present several experiments to validate the performance of the proposed strategy, both in $2 \mathrm{D}$ and $3 \mathrm{D}$. The goal of these examples is to show the robustness of the methodology to capture complex crack patterns with a coarse and fixed background mesh during all the simulation, while the discretization is dynamically refined along cracks.

In all two-dimensional examples, plane strain conditions are assumed for the equilibrium equation. The restoring of stiffness under compression in equation (1c) is only necessary for the branching test in Subsection 5.3. In the other examples, $g(d)=(1-d)^{2}$ in the whole domain. Preexisting cracks which are described as smeared damage bands are introduced by an initial history field variable, $\mathcal{H}_{0}^{+}$, following Borden et al [4]. The tolerance for convergence of the damage field $d$ in the staggered scheme is fixed to $10^{-2}$. The parameters in the Nitsche's formulation for both equations are $\alpha_{\mathrm{E}}=\alpha_{\mathrm{D}}=100$.

\subsection{Shear test}

Consider a square plate with a precrack at mid-height, which is fixed on its bottom edge and has imposed horizontal displacement $u_{D}$ on its top edge, as shown in Fig. 11. Following Ambati et al [1], the material parameters

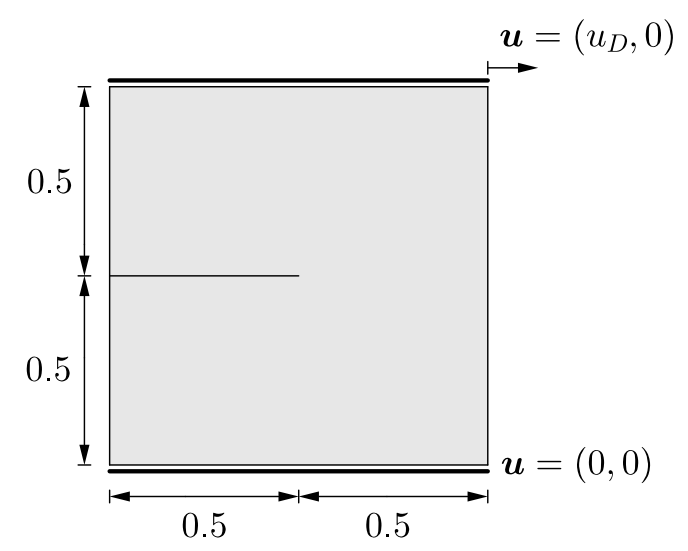

Fig. 11: Shear test. Domain and boundary conditions. Dimensions in mm. 

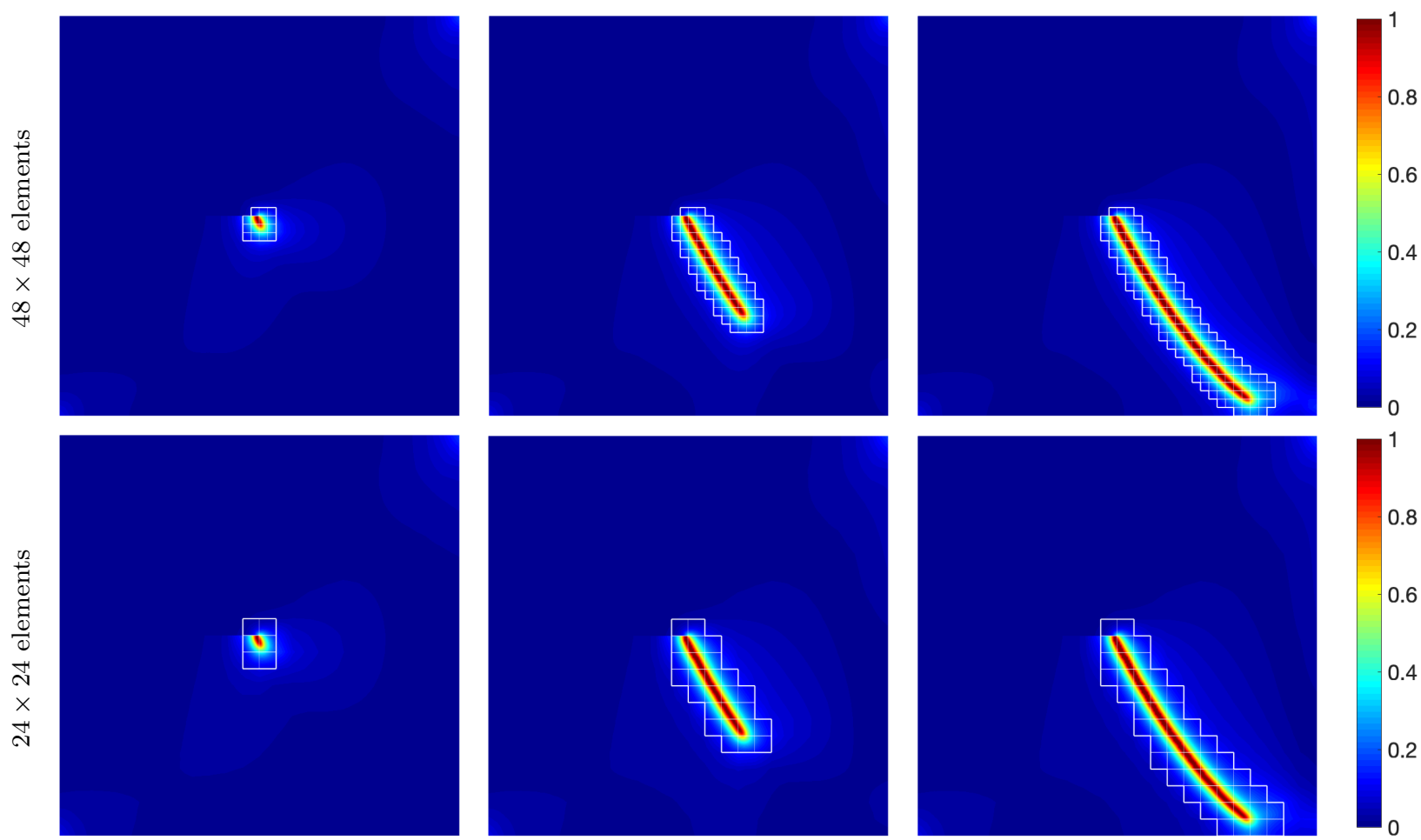

$u_{D}=0.010 \mathrm{~mm}$

$u_{D}=0.013 \mathrm{~mm}$

$u_{D}=0.020 \mathrm{~mm}$

Fig. 12: Shear test. Damage field at imposed displacements $u_{D}$, for degree $p=1$ and refinement value $d^{*}=0.2$.

are $E=210 \mathrm{GPa}, \nu=0.3$ and $G_{C}=2.7 \cdot 10^{-3} \mathrm{kN} / \mathrm{mm}$. We use $l=0.015 \mathrm{~mm}$. The loading process takes increments $\Delta u_{D}=10^{-4} \mathrm{~mm}$ and the degree of approximation is $p=1$.

First, we compare the solution obtained applying the refinement strategy with a reference solution computed on a globally refined mesh. The problem is solved on uniform quadrilateral background meshes with $48 \times$ $48,24 \times 24$ and $12 \times 12$ elements, with respective refinement factors $m=5,10$ and 20 . The reference solution is computed on a mesh with $240 \times 240$ elements. Note that all discretizations have the same element size along the crack. The four elements surrounding the initial crack tip are refined in the preprocess for all discretizations.

Fig. 12 shows the damage field at three load steps for the discretizations with initial meshes of $48 \times 48$ and $24 \times 24$ elements, for refinement value $d^{*}=0.2$. Refined elements are highlighted with white edges. As the crack propagates, a narrow band of elements along the crack is refined.

The agreement between the reference solution, using a globally refined mesh, and the considered discretizations with automatic refinement can be seen in the contour plots in Fig. 13. The corresponding loaddisplacement curves are plotted in Fig. 14. For the mesh with $12 \times 12$ elements, the crack path obtained differs from the other ones. This can be explained by the in- accuracy of the background mesh to resolve the mechanical problem in the nonrefined region. The resulting crack path for the reference mesh is very similar to those for the $48 \times 48$ and the $24 \times 24$ meshes.

We can conclude that a very local refinement along cracks is enough to capture the solution. This example demonstrates the robustness of the strategy to automatically adapt the discretization. Also, the refinement criterion based on the value of $d$ performs as expected.

Now, we study the influence of the refinement factor $d^{*}$, this is, the threshold value activating the refinement of elements. Consider the discretization with $24 \times 24$ elements and refinement factor $m=10$. For refinement values $d^{*}=0.1,0.2,0.5$ and 0.7 , we plot the load-displacement curves in Fig. 15. The kinks coincide with the refinement of elements, this is, they are corrections once the accuracy of the discretization increases. According to the results, a value for $d^{*}$ between 0.1 and 0.2 gives accurate results, with a narrow band of refinement along the crack.

The saving in degrees of freedom for adaptive discretizations is also remarkable. Table 1 lists the number of degrees of freedom for each one of the discretizations at the beginning and at the end of the simulation, for refinement values $d^{*}=0.1$ and 0.2 . With the proposed strategy, we are able to obtain accurate results with about $10-15 \%$ of degrees of freedom of the globally 

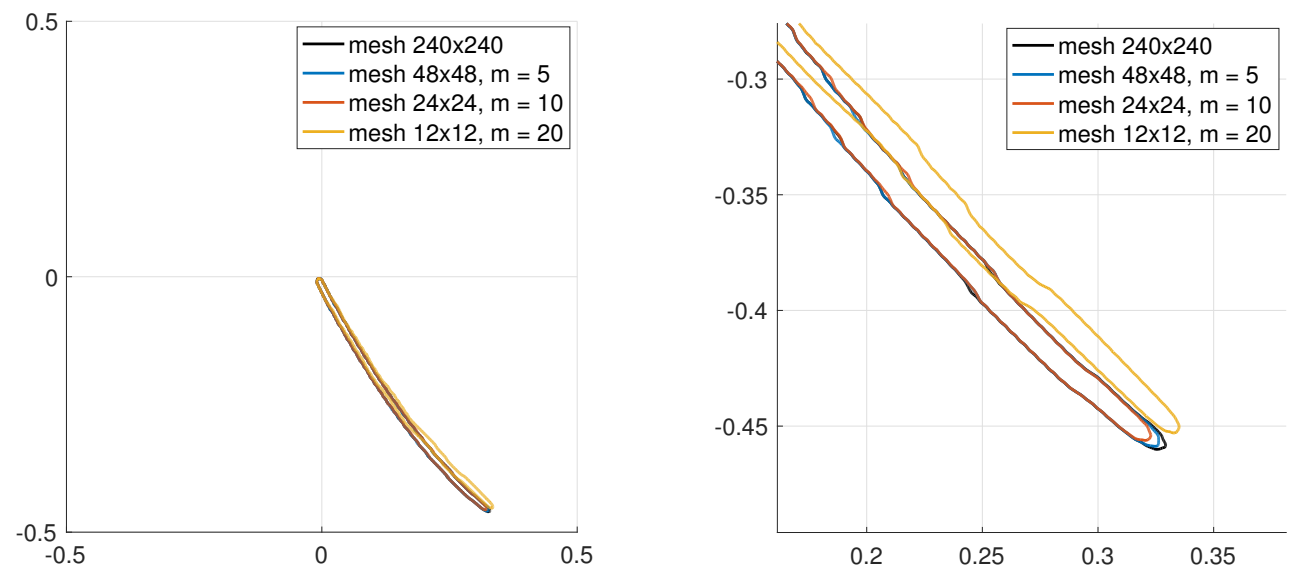

Fig. 13: Shear test. Contour plot for damage value $d=0.9$, at imposed displacement $u_{D}=0.020 \mathrm{~mm}$ with refinement value $d^{*}=0.2$. Zoom at the crack tip on the right.

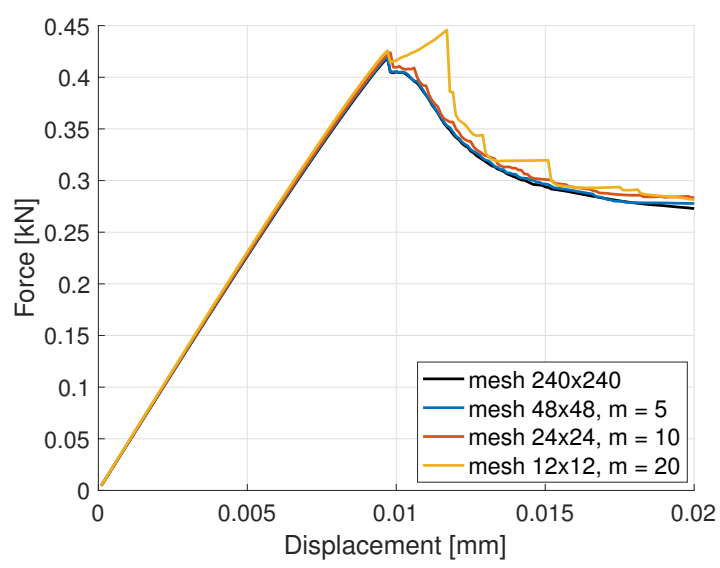

Fig. 14: Shear test. Load-displacement curve, for degree of approximation $p=1$ and refinement value $d^{*}=0.2$.

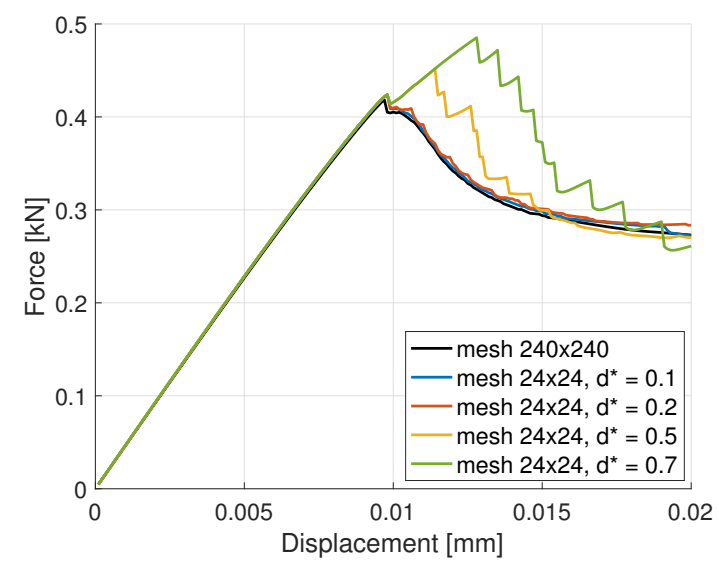

Fig. 15: Shear test. Load-displacement curve for refinement factors $d^{*}$. Mesh with $24 \times 24$ elements, refinement factor $m=10$ and degree $p=1$.
Table 1: Shear test. Degrees of freedom of the equilibrium problem for the various discretizations

\begin{tabular}{llllll}
\hline Mesh & $m$ & $d^{*}$ & Initial \#dof & Final \#dof & $\%$ \\
\hline $240 \times 240$ & - & - & 115438 & 115438 & 100 \\
\hline $48 \times 48$ & 5 & 0.1 & 4904 & 17626 & 15.3 \\
& & 0.2 & 4904 & 12804 & 11.1 \\
\hline $24 \times 24$ & \multirow{2}{*}{10} & 0.1 & 2074 & 17108 & 14.8 \\
& & 0.2 & 2074 & 11738 & 10.2 \\
\hline $12 \times 12$ & 20 & 0.1 & 3698 & 22350 & 19.4 \\
& & 0.2 & 3698 & 16722 & 14.5 \\
\hline
\end{tabular}

refined mesh. Notice that for the coarser mesh, with $12 \times 12$ elements, the percentage of degrees of freedom is higher than for the other two adaptive discretizations. This is due to obtaining a wider refined zone in the mesh. Depending on the accuracy needed, one has to find a compromise between the background mesh, the refinement factor $m$ and the refinement value $d^{*}$ to attain a feasible computational cost.

\subsection{Notched plate with a hole}

This test was proposed by Ambati et al [1]. With this example, we want to test the performance of our adaptivity approach for a nonstructured mesh.

A precracked plate with a noncentered hole and two pins is subjected to vertical displacements, as shown in Fig. 16. The plate is fixed on the lower pin and has imposed vertical displacements, $u_{D}$, on the upper pin. The material parameters are $E=6 \mathrm{GPa}, \nu=0.22$ and $G_{C}=2.28 \cdot 10^{-3} \mathrm{kN} / \mathrm{mm}$. We use $l=0.5 \mathrm{~mm}$, and 


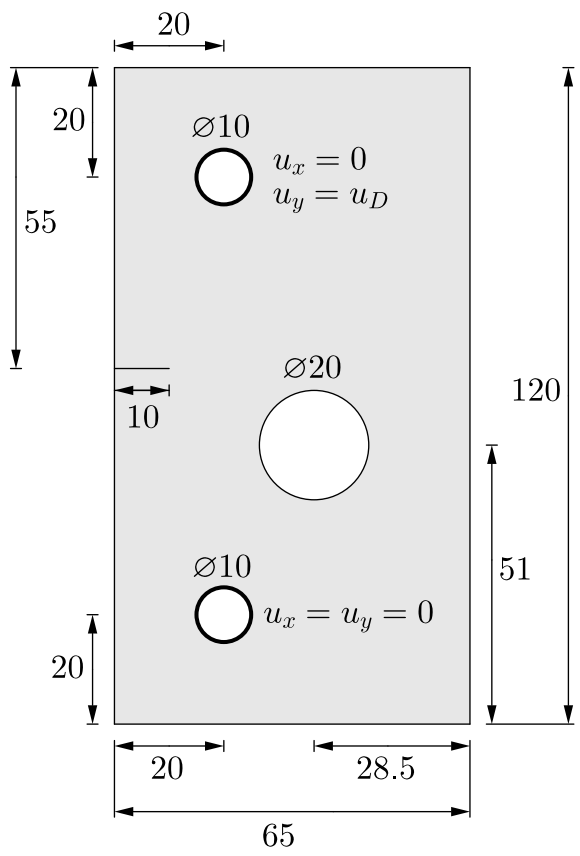

Fig. 16: Plate with a hole. Domain and boundary conditions. Dimensions in $\mathrm{mm}$.

take load increments of $\Delta u_{D}=10^{-3} \mathrm{~mm}$. We consider a quadrilateral mesh with element size $h \simeq 5$ $\mathrm{mm}$, necessarily nonstructured to fit the geometry of the specimen, with degree of approximation $p=4$ and refinement factor $m=10$. Recall that the geometry of the domain is described by this background mesh during all the simulation. Elements containing the initial crack are refined from the beginning. Then, refinement is triggered by threshold value $d^{*}=0.2$.

Fig. 17 displays the crack pattern for some load steps and Fig.18, the corresponding load-displacement curve. The crack horizontally propagates down to the hole and develops on the other side as elements are accordingly refined. As can be clearly observed in the load-displacement curve, these propagations are quite abrupt as expected in brittle fracture. This behavior corroborates the need for applying the refinement criterion at every staggered iteration. Again, the obtained results manifest the good performance of the strategy, here for a higher degree of approximation and a more complex scenario.

\subsection{Branching test}

This test was first proposed in Muixí et al [19]. It offers a setting for crack branching in the quasi-static regime, with no heterogeneities in the material.

Consider a square plate in the domain $[-1,1]^{2} \mathrm{~mm}^{2}$, with a precrack at mid-height as shown in Fig. 19. The plate is clamped on its right edge and has imposed vertical displacements on its top and bottom edges, following the parabolic function $f(x)=u_{D}(x-1)^{2} / 8$.

The material parameters are $E=20 \mathrm{GPa}, \nu=0.3$ and $G_{C}=8.9 \cdot 10^{-5} \mathrm{kN} / \mathrm{mm}$. The numerical lengthscale parameter is $l=0.01 \mathrm{~mm}$ and the refinement value is $d^{*}=0.2$. The loading process takes increments $\Delta u_{D}=5 \cdot 10^{-5} \mathrm{~mm}$. The specimen is discretized into a quadrilateral uniform mesh of $45 \times 45$ elements and refinement factor $m=15$, with degree of approximation $p=1$.

As depicted in Fig. 20, the initial crack propagates horizontally and branches before reaching the right edge. The load-displacement curve is in Fig. 21. Due to the bending caused by the imposed displacements, the damage field reaches the refinement value $d^{*}$ in elements on the left corners of the domain. The adaptive strategy enables to capture the branching maintaining the symmetry of the solution.

With this example, we can illustrate the role of the hybrid condition in equation (1c) of the model. If elastic stiffness is not restored under compression, we observe interpenetration of faces near the branching point when branches propagate. In Fig. 22 we plot the deformed mesh at load step $u_{D}=0.075 \mathrm{~mm}$ in two cases: taking $g(d)=(1-d)^{2}$ in all the domain, and restoring $g(d)$ to 1 in compressed regions as stated in (1c). Although we obtain crack branching in both cases, a slight interpenetration of faces can be observed if the hybrid condition is not implemented.

\subsection{Plate with multiple cracks}

This test is inspired by the multiple-cracked plate test by Budyn et al [6]. It exemplifies a case for which an automatic refinement of the discretization is crucial.

We consider a square plate occupying the domain $[0,2]^{2} \mathrm{~mm}^{2}$, with six pre-existing cracks, loaded with prescribed displacements as shown in Fig. 23. The tips of the initial cracks are reported in Table 2. The parameters are $E=20 \mathrm{GPa}, \nu=0.3, G_{C}=10^{-3} \mathrm{kN} / \mathrm{mm}$ and $l=0.012 \mathrm{~mm}$, with applied increments of $\Delta u_{D}=$ $5 \cdot 10^{-5} \mathrm{~mm}$. We use a uniform mesh of $40 \times 40$ elements, with degree of approximation $p=2$, refinement factor $m=10$ and $d^{*}=0.2$.

In this case, cracks propagate coalescing between them. Applying the proposed strategy, we are able to capture crack propagation until the piece has broken into four independent pieces. Fig. 24 shows the evolution of the crack pattern for some imposed displacements; each one of them corresponds to an abrupt growth of one of the cracks. The respective load-displacement 


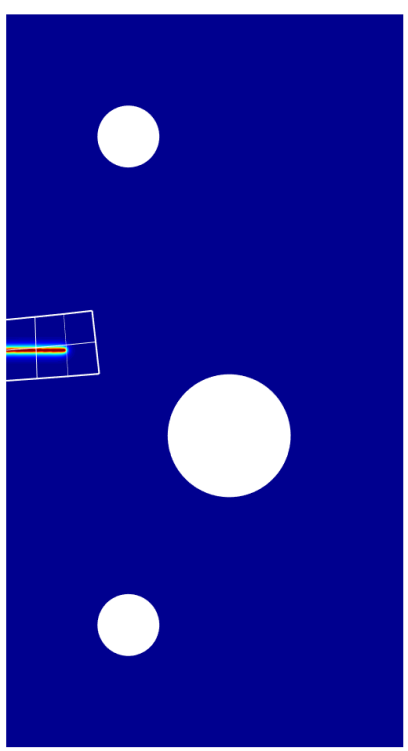

$u_{D}=0 \mathrm{~mm}$

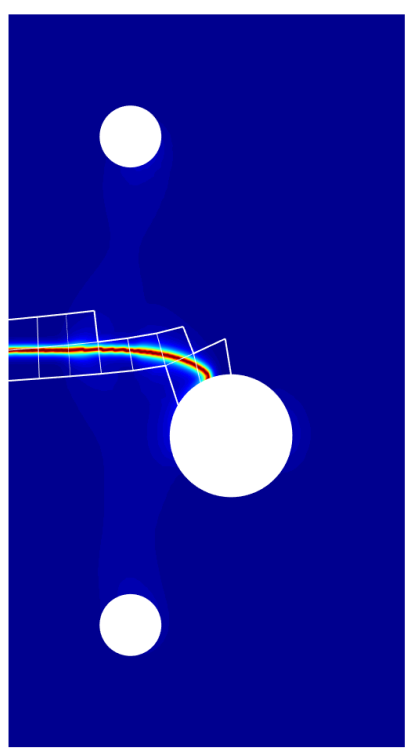

$u_{D}=0.4 \mathrm{~mm}$

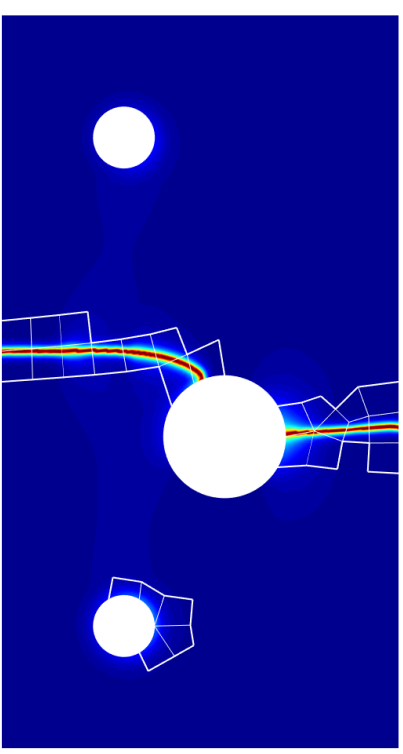

$u_{D}=1.05 \mathrm{~mm}$

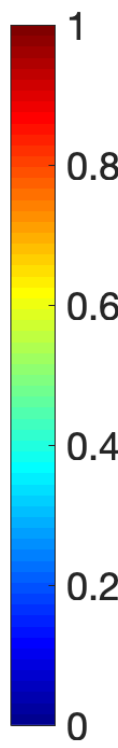

0.8

0.6

0.4

0.2

Fig. 17: Plate with a hole. Damage field at imposed displacements $u_{D}$. Nonstructured mesh with element size $h \simeq 5$ $\mathrm{mm}$, degree of approximation $p=4$ and refinement factor $m=10$.

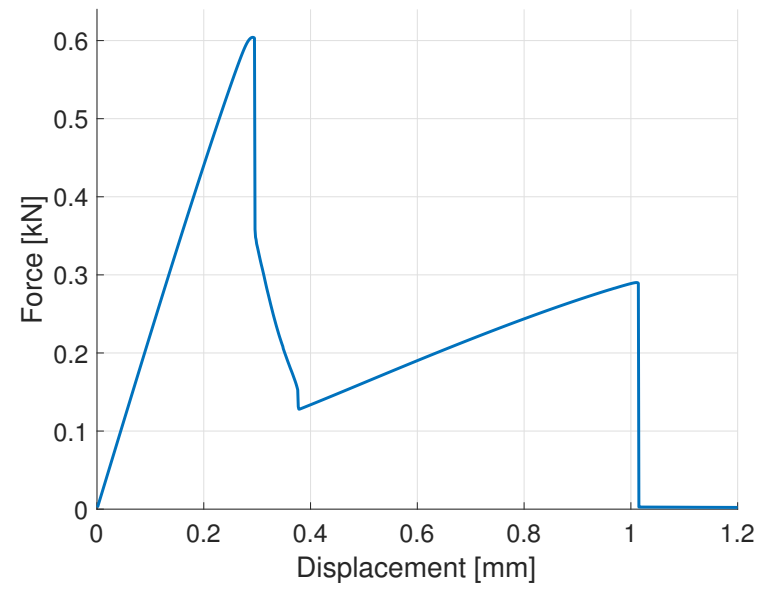

Fig. 18: Plate with a hole. Load-displacement curve, for degree of approximation $p=4$ and refinement factor $m=10$.

Table 2: Multiple cracks test. Tip coordinates for the initial cracks in the domain $[0,2]^{2} \mathrm{~mm}^{2}$.

\begin{tabular}{cll}
\hline Crack & $P_{1}(\mathrm{~mm})$ & $P_{2}(\mathrm{~mm})$ \\
\hline 1 & $(0.5,1.5)$ & $(0.6,1.55)$ \\
2 & $(1,1.1)$ & $(1,1.5)$ \\
3 & $(1.4,1.5)$ & $(1.5,1.55)$ \\
4 & $(0.5,0.9)$ & $(0.7,0.9)$ \\
5 & $(0.5,0.5)$ & $(0.6,0.45)$ \\
6 & $(1.2,0.5)$ & $(1.5,0.6)$ \\
\hline
\end{tabular}

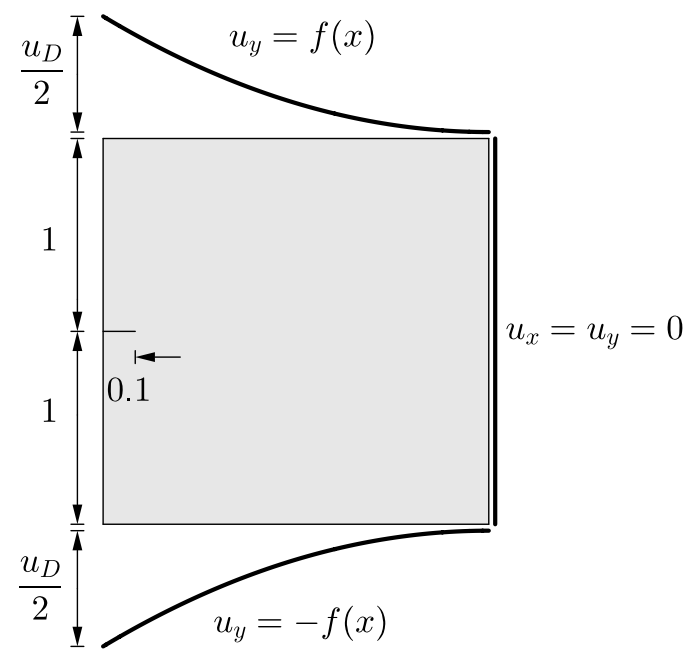

Fig. 19: Branching test. Domain and boundary conditions. Dimensions in $\mathrm{mm}$.

curves, for both the horizontal and the vertical loads, $F_{x}$ and $F_{y}$, are in Fig. 25. Notice that all abrupt propagations coincide with a force drop in the load force. The piece loses the horizontal stiffness once a vertical crack crosses the whole plate, at $u_{D}=0.015 \mathrm{~mm}$. The vertical stiffness is also lost at the end of the process, when the piece is completely broken apart. The complete evolution of the cracks propagation and of the automatic refinement can be seen in the YouTube video [18].

This example highlights the reliability of the strategy for cases in which the refined zones are scattered 


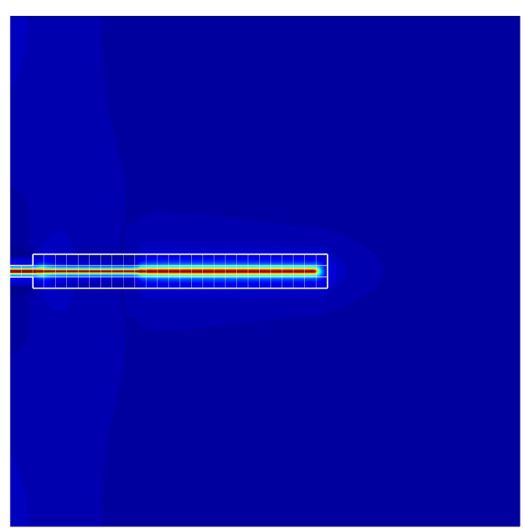

$u_{D}=0.02 \mathrm{~mm}$

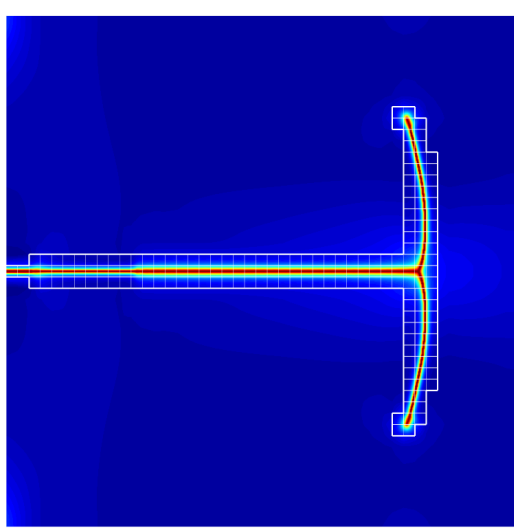

$u_{D}=0.059 \mathrm{~mm}$

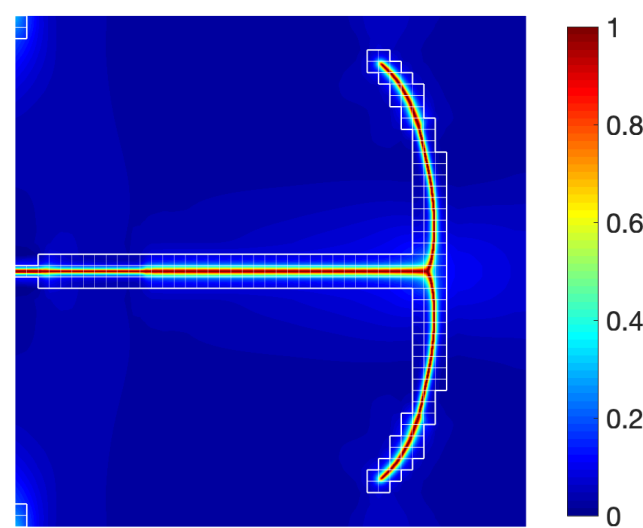

$u_{D}=0.095 \mathrm{~mm}$

Fig. 20: Branching test. Damage field at different load steps. Degree of approximation $p=1$ and refinement factor $m=15$.

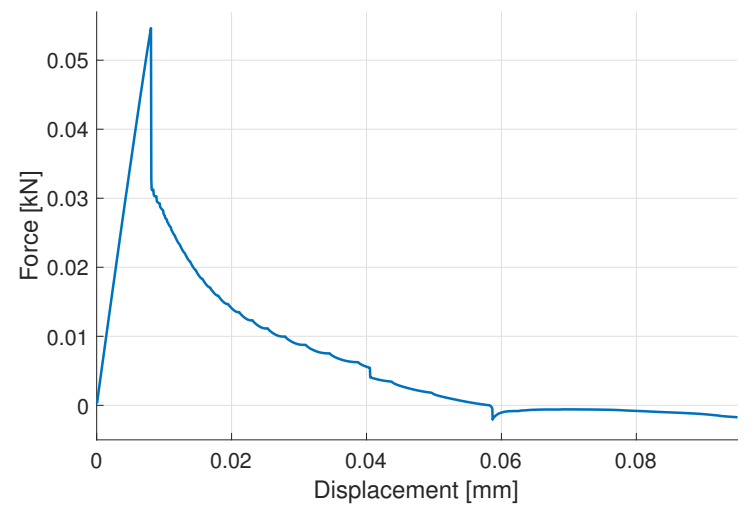

Fig. 21: Branching test. Load-displacement curve, for degree $p=1$ into a $45 \times 45$ mesh with refinement factor $m=15$.

in the domain. The union of refined groups of elements and the corresponding rearrangement of interface faces, where Nitsche's method is applied, properly capture the fracture process.

\subsection{Twisting crack in a 3D beam}

Finally, we test the performance of the strategy for a $3 \mathrm{D}$ setting. Consider a beam with square section $\Omega=$ $[0,125] \times[0,25] \times[0,25] \mathrm{mm}^{3}$ as shown in Fig. 26. The piece has two inclined notches with opposite angles, on faces $\{y=0 \mathrm{~mm}\}$ and $\{y=25 \mathrm{~mm}\}$. The beam is clamped on $\{x=0 \mathrm{~mm}\}$ and has imposed displacements in the $x$ direction on the face $\{x=125 \mathrm{~mm}\}$. Because of the orientation of the notches, this example cannot be reduced to a $2 \mathrm{D}$ approximate configuration.
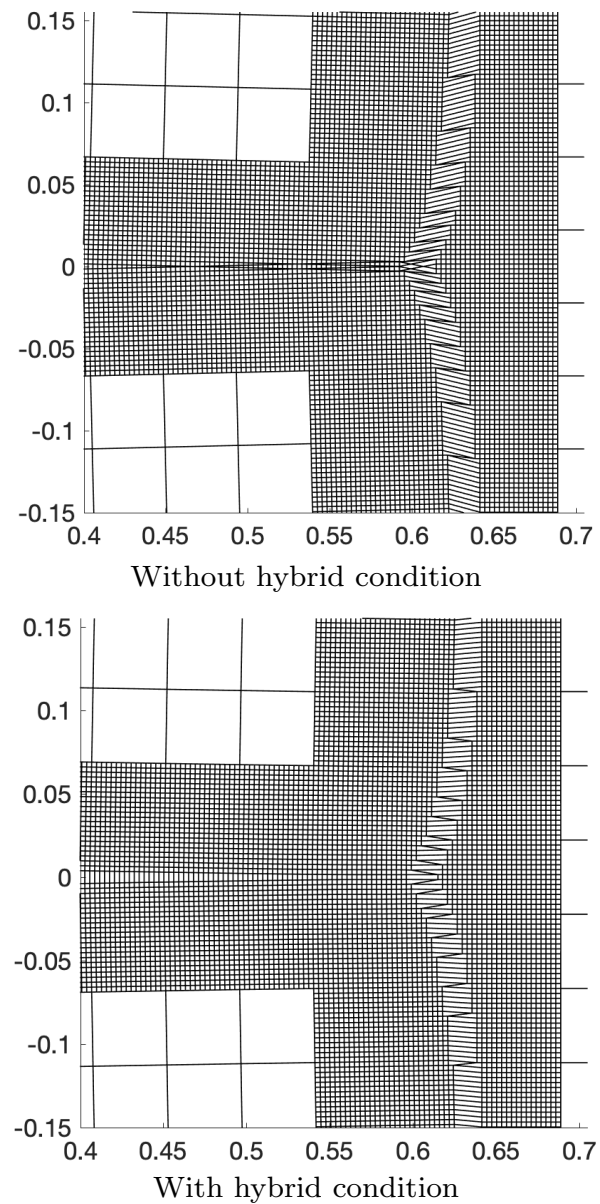

Fig. 22: Branching test. Zoom of the deformed discretization at the branching point, with and without imposing the hybrid condition in equation (1c), at load step $u_{D}=0.075 \mathrm{~mm}$ for a mesh with $45 \times 45$ elements, $m=15$ and $p=1$. 


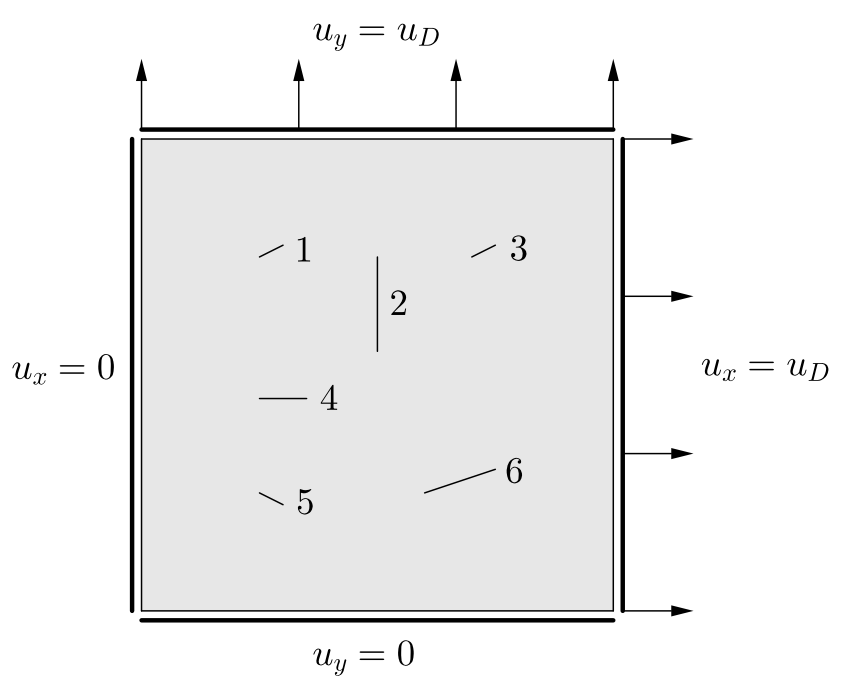

Fig. 23: Multiple cracks test. Domain and boundary conditions.

The parameters are $E=32 \mathrm{GPa}, \nu=0.25, G_{C}=$ $1.6 \cdot 10^{-4} \mathrm{kN} / \mathrm{mm}$ and $l=2 \mathrm{~mm}$. The loading process takes increments $\Delta u_{D}=5 \cdot 10^{-4} \mathrm{~mm}$.

We consider a uniform mesh of hexahedra with element size $h=5 \mathrm{~mm}$. The degree of approximation is $p=2$ and the refinement factor is $m=5$. Refinement is activated with threshold value $d^{*}=0.2$. We model the initial notches as damage bands and refine the elements containing them in the preprocess.

The resulting damage field is shown in Fig. 27. Inital cracks coalesce, with a twisting to match the opposite inclinations of the notches. In Fig. 28 we plot the crack path as seen from the exterior faces of the beam. We observe the expected symmetry with respect of rotations of the piece around the $x$-axis. The mesh is refined in a narrow band containing the crack. The load-displacement curve is in Fig. 29 and indicates that the specimen is completely broken at a single load step.

This example illustrates the ability of the strategy to simulate cracks also in 3D, where more computational resources are needed and adaptivity is a key part of the procedure.

\section{Conclusions}

A novel adaptive refinement strategy for phase-field models of brittle fracture has been presented. The strategy consists in defining two types of elements, standard and refined, and imposing continuity between different discretization spaces in weak form by means of Nitsche's method. Weak continuity implies that there are no hanging nodes nor transition elements. The discretization is automatically refined in narrow bands along cracks and shows no refinement spreading. The initial background mesh is kept constant in the whole computation, i.e., no adaptive mesh generation is needed.

Regarding the choice of the Nitsche's parameter, the formulation is stable for a wide range of values. A lower bound of this parameter may be rigorously obtained by solving an eigenvalue problem. However, according to our experience, finding a suitable value by numerical experimentation is rather straightforward.

The accuracy and robustness of the strategy has been illustrated through several numerical examples, both in 2D and 3D. The method has been tested for complex scenarios, such as crack branching and coalescence, for high orders of approximation and for refinement factors up to $m=20$. In all the cases, the analysis is successfully carried out without any a priori information about the crack path required for the definition of the background mesh.

The strategy can be easily added to an existing finite element code for phase-field and can be analogously applied to other phase-field models of fracture.

Acknowledgements This work was supported by the Agència de Gestió d'Ajuts Universitaris i de Recerca training grant FI-DGR 2017, the DAFOH2 project (Ministerio de Ciencia e Innovación, MTM2013-46313-R) and the Departament d'Innovació, Universitats i Empresa, Generalitat de Catalunya (2017-SGR-1278).

\section{References}

1. Ambati, M., Gerasimov, T., De Lorenzis, L.: A review on phase-field models of brittle fracture and a new fast hybrid formulation. Comput Mech 55, 383-405 (2015)

2. Annavarapu, C., Hautefeuille, M., Dolbow, J.: A robust Nitsche's formulation for interface problems. Comput Methods Appl Mech Eng s 225-228, 44-54 (2012)

3. Becker, R., Hansbo, P., Stenberg, R.: A finite element method for domain decomposition with non-matching grids. ESAIM: Math Model Numer Anal 37(2), 209-225 (2010)

4. Borden, M.J., Verhoosel, C.V., Scott, M.A., Hughes, T.J.R., Landis, C.M.: A phase-field description of dynamic brittle fracture. Comput Methods Appl Mech Eng 217-220, 77-95 (2012)

5. Bourdin, B., Francfort, G.A., Marigo, J.J.: Numerical experiments in revisited brittle fracture. J Mech Phys Solids 48, 797-826 (2000)

6. Budyn, E., Zi, G., Moës, N., Belytschko, T.: A method for multiple crack growth in brittle materials without remeshing. Int J Numer Methods Eng 61(10), 1741-1770 (2004)

7. Fernández-Méndez, S., Huerta, A.: Imposing essential boundary conditions in mesh-free methods. Comput Methods Appl Mech Eng 193(12-14), 1257-1275 (2004)

8. Geelen, R.J., Liu, Y., Dolbow, J., Rodríguez-Ferran, A.: An optimization-based phase-field method for continuous-discontinuous crack propagation. Int $\mathrm{J} \mathrm{Nu}-$ mer Methods Eng 116(1), 1-20 (2018) 


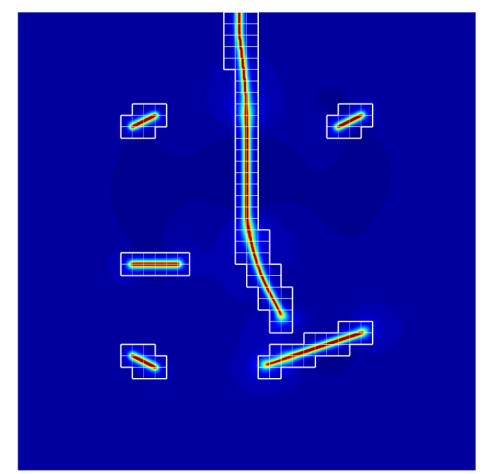

$u_{D}=0.0125 \mathrm{~mm}$

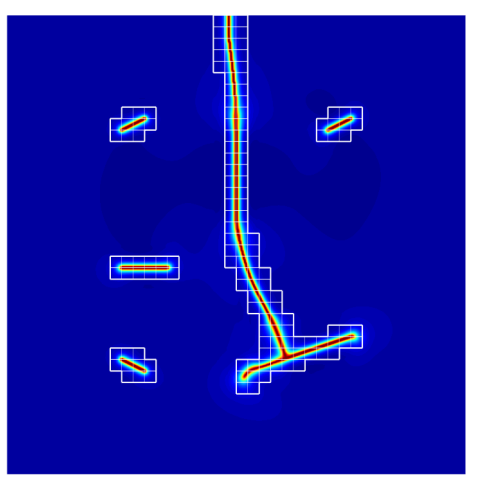

$u_{D}=0.0137 \mathrm{~mm}$

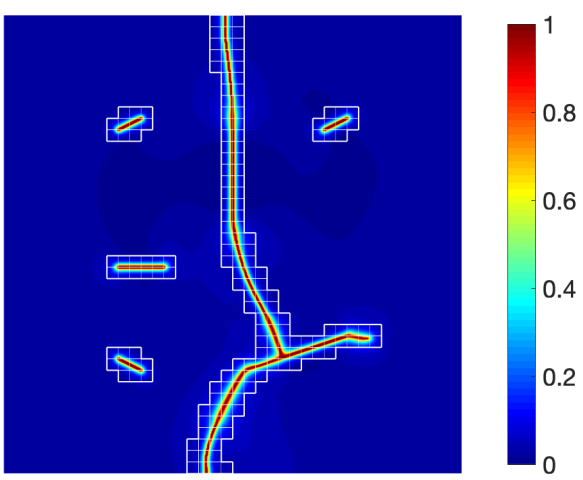

$u_{D}=0.0150 \mathrm{~mm}$

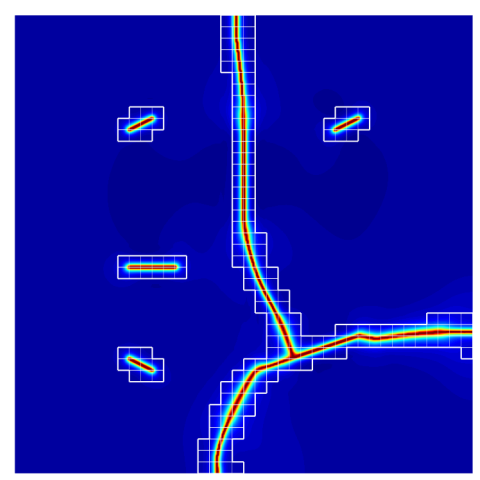

$u_{D}=0.0195 \mathrm{~mm}$

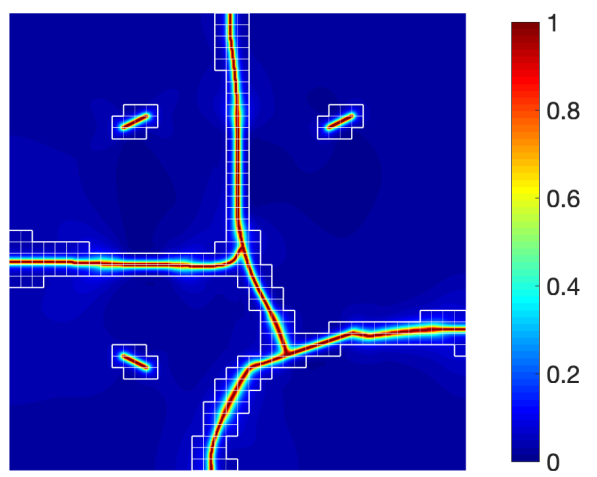

$u_{D}=0.0271 \mathrm{~mm}$

Fig. 24: Multiple cracks test. Damage field at different load steps. Degree of approximation $p=2$, refinement factor $m=10$ and $d^{*}=0.2$ on a $45 \times 45$ quadrilateral mesh.

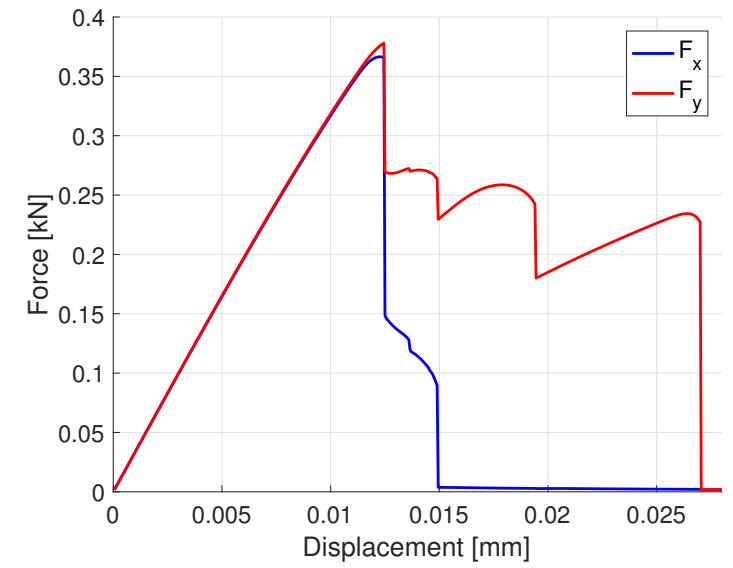

Fig. 25: Multiple cracks test. Load-displacement curve. Degree $p=2, m=10$ and $d^{*}=0.2$ on a $45 \times 45$ quadrilateral mesh.

9. Geelen, R.J., Liu, Y., Hu, T., Tupek, M., Dolbow, J.: A phase-field formulation for dynamic cohesive fracture. Comput Methods Appl Mech Eng 348, 680-711 (2019)

10. Griebel, M., Schweitzer, M.A.: A particle-partition of unity method. Part V: Boundary conditions. In Stefan Hildebrandt and Hermann Karcher, editors, Geometric Analysis and Nonlinear Partial Differential Equations,

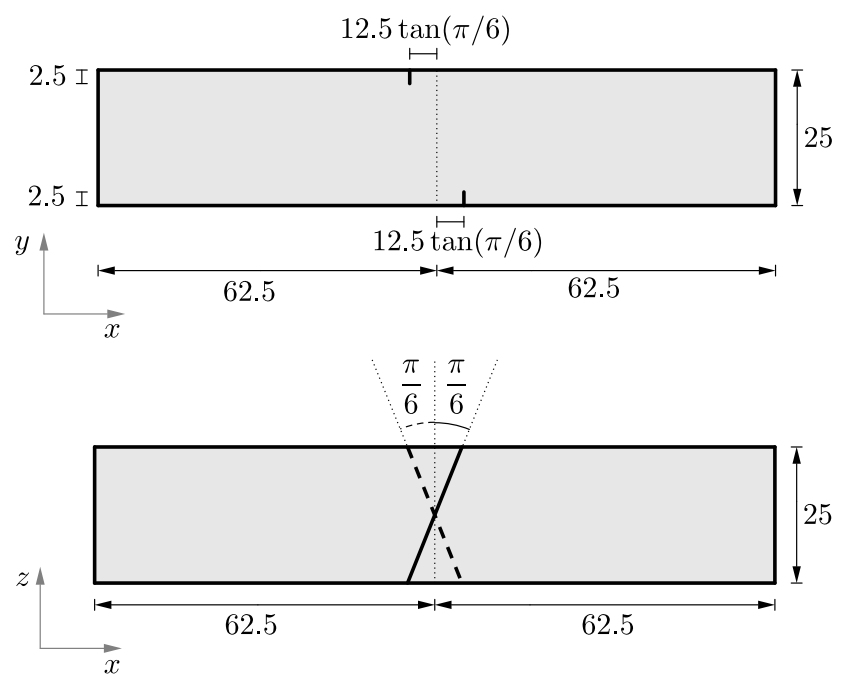

Fig. 26: Twisting crack test. Geometry seen from the plane $\{z=0\}$ and from $\{y=25\}$. Continuous line indicatess the notch on $\{y=25\}$ and the dashed line, the notch on $\{y=0\}$. Dimensions in mm, angles in rad.

Springer, Berlin pp. 519-542 (2003)

11. Hansbo, P.: Nitsche's method for interface problems in computational mechanics. GAMM-Mitteilungen 28(2), 


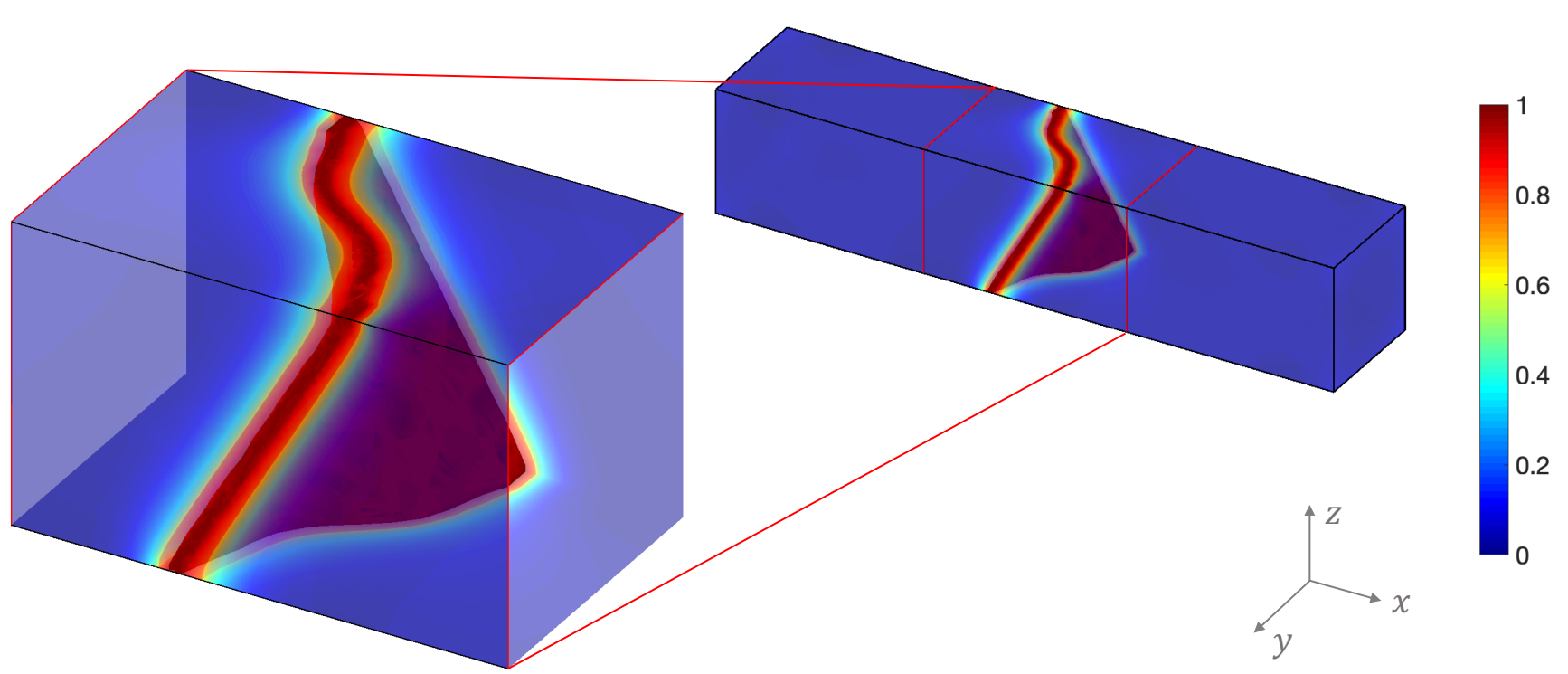

Fig. 27: Twisting crack test. Crack path obtained at imposed displacement $u_{D}=0.066 \mathrm{~mm}$. The piece is discretized into a hexahedral mesh with $h=5 \mathrm{~mm}$, degree $p=2$, refinement factor $m=5$ and $d^{*}=0.2$.

Front view $\{y=25 \mathrm{~mm}\}$

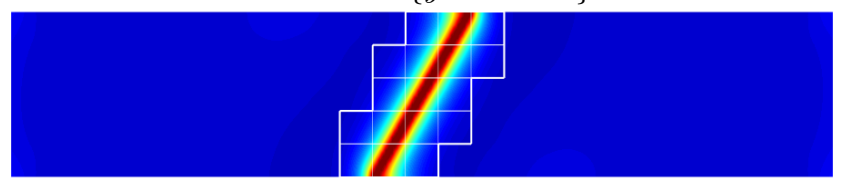

Top view $\{z=25 \mathrm{~mm}\}$

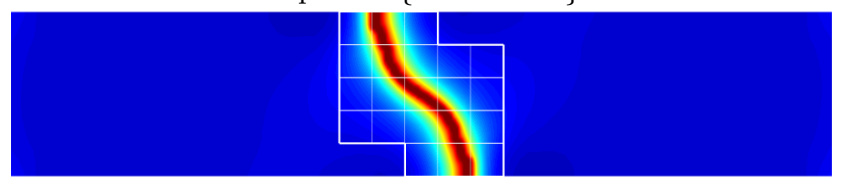

Rear view $\{y=0 \mathrm{~mm}\}$

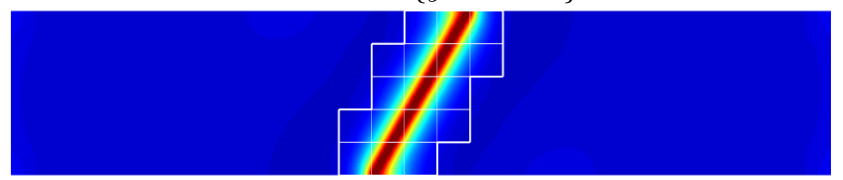

Bottom view $\{z=0 \mathrm{~mm}\}$

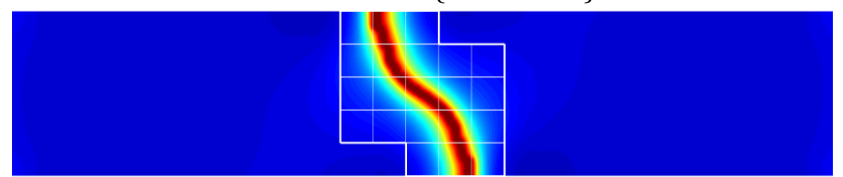

Fig. 28: Twisting crack test. Crack path seen from the exterior faces of the piece for $u_{D}=0.066 \mathrm{~mm}$. Degree $p=2$ and refinement value $d^{*}=0.2$.

183-206 (2005)

12. Hennig, P., Ambati, M., De Lorenzis, L., Kästner, M.: Projection and transfer operators in adaptive isogeometric analysis with hierarchical B-splines. Comput Methods Appl Mech Eng 334, 313-336 (2018)

13. Hennig, P., Müller, S., Kästner, M.: Bézier extraction and adaptive refinement of truncated hierarchical NURBS. Comput Methods Appl Mech Eng 305, 316-339 (2016)

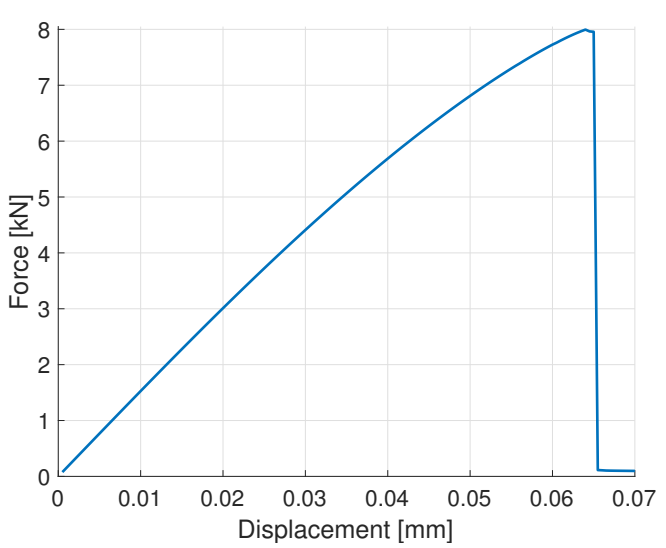

Fig. 29: Twisting crack test. Load-displacement curve, for a uniform mesh with $h=5 \mathrm{~mm}$ and degree $p=2$, refinement factor $m=5$ and refinement value $d^{*}=0.2$.

14. La Spina, A., Giacomini, M., Huerta, A.: Hybrid coupling of CG and HDG discretizations based on Nitsche's method. To appear in Computational Mechanics (2019)

15. Lo, Y.S., Borden, M.J., Ravi-Chandar, K., Landis, C.M.: A phase-field model for fatigue crack growth. J Mech Phys Solids 132, 103684 (2019)

16. Miehe, C., Hofacker, M., Welschinger, F.: A phase-field model for rate-independent crack propagation: robust algorithmic implementation based on operator splits. Comput Methods Appl Mech Eng 199, 2765-2778 (2010)

17. Miehe, C., Welschinger, F., Hofacker, M.: Thermodynamically consistent phase-field models of fracture: variational principles and multi-field FE implementations. Int J Numer Methods Eng 83, 1273-1311 (2010)

18. Muixí, A., Fernández-Méndez, S., Rodríguez-Ferran, A.: Phase-field solution of brittle fracture with adaptive refinement. https://youtu.be/FiQQe6UpenI (2019) 
19. Muixí, A., Rodríguez-Ferran, A., Fernández-Méndez, S.: A Hybridizable Discontinuous Galerkin phase-field model for brittle fracture with adaptive refinement. Int J Numer Methods Eng (2019)

20. Nagaraja, S., Elhaddad, M., Ambati, M., Kollmannsberger, S., De Lorenzis, L., Rank, E.: Phase-field modeling of brittle fracture with multi-level $h p$-FEM and the finite cell method. Comput Mech 6 (2019)

21. Nitsche, J.: Über ein variationsprinzip zur lösung von Dirichlet-problemen bei verwendung von teilr äumen, die keinen randbedingungen unterworfen sind. Abh Math Se Univ 36(1), 9-15 (1971)

22. Noii, N., Aldakheel, F., Wick, T., Wriggers, P.: An adaptive global-local approach for phase-field modeling of anisotropic brittle fracture. Accepted November 2019, Comput Methods Appl Mech Eng https://arxiv.org/pdf/1905.07519.pdf (2019)

23. Patil, R.U., Mishra, B.K., Singh, I.V.: A local moving extended phase field method (LMXPFM) for failure analysis of brittle fracture. Comput Methods Appl Mech Eng 342, 674-709 (2018)

24. Tamayo-Mas, E., Rodríguez-Ferran, A.: A medial-axisbased model for propagating cracks in a regularised bulk. Int J Numer Methods Eng 101(7), 489-520 (2015)

25. Wu, J.Y., Nguyen, V.P., Nguyen, C.T., Sutula, D., Bordas, S., Sinaie, S.: Phase field modelling of fracture. Adv Appl Mech 53 (2019) 